\title{
Computed Tomography Evaluation Of Normal Canine Abdominal Lymph Nodes: Retrospective Study Of Size And Morphology According To Body Weight And Age In 45 Dogs
}

\section{Simone Teodori}

Roma Sud Veterinary Clinic

\section{Giovanni Aste}

Universita degli Studi di Teramo Facolta di Medicina Veterinaria

Roberto Tamburro ( $\nabla$ rtamburro@unite.it)

Universita degli Studi di Teramo https://orcid.org/0000-0001-6198-163X

Antonio Morselli Labate

Biostatistics

Francesco Simeoni

Universita degli Studi di Teramo Facolta di Medicina Veterinaria

Massimo Vignoli

Universita degli Studi di Teramo Facolta di Medicina Veterinaria

\section{Research article}

Keywords: computed tomography, lymph nodes, dog

Posted Date: March 11th, 2020

DOI: https://doi.org/10.21203/rs.3.rs-16758/v1

License: (c) (i) This work is licensed under a Creative Commons Attribution 4.0 International License.

Read Full License

Version of Record: A version of this preprint was published at Veterinary Sciences on March 7th, 2021. See the published version at https://doi.org/10.3390/vetsci8030044. 


\section{Abstract}

\section{Background}

The morphological characteristics of the largest lymphatic vessels and lymph nodes of the body have been described through ultrasonography, although food and gas in the gastrointestinal tract can often have negative effects on the response of small abdominal structures. The aim of the study was to describe the size of normal abdominal lymph nodes (ALs) in dogs affected by disease, not including lymphadenomegaly or lymphadenopathy, and divided according to body weight and age. The ALs studied included the jejunal, medial iliac, portal, gastric, splenic, and pancreaticoduodenal lymph nodes.

Results

Multiple statistical analyses among the three groups showed that all measurements of the ALs increased according to body weight changes $(P<0.05)$. The most reliable values were the volume measurements $(P$ $<0.001)$ compared to the length, thickness and width. A single analysis between just two groups showed different values $(P>0.1)$. Mixed results emerged from a comparison of weight categories and age; only the jejunal lymph nodes showed a possible correlation $(P<0.1)$. Other characteristics (shape, attenuation and enhancement) are subsequently reported.

\section{Conclusions and Clinical Relevance}

The resulting data can be used to categorize CT measurements of normal ALs displayed based on the body weight and age of the subjects. This study was able to detect an objective parameter of normalcy that can serve as a reference for the evaluation of infectious or neoplastic events.

\section{Background}

The largest lymphatic vessels and lymph nodes of the body are described regionally according to the following categories: The head and neck, thoracic limb, thorax, abdominal and pelvic walls, genital organs, abdominal viscera and pelvic limb. The abdomen and pelvis, such as the chest, can be divided into a parietal group and a visceral group. The parietal group includes the lymph-node centre of the abdominal and pelvic walls: the lumbar, iliosacral and iliofemoral centres. The visceral group is subdivided into subgroups that apply to specific organs: celiac, cranial and caudal mesenteric [6]. For many of these lymph nodes, morphological characteristics have been described through ultrasonography, although food and gas in the gastrointestinal tract can often have negative effects on the response of small abdominal structures $[1,3,4,13,15,16,21]$. There are studies for tracheobronchial, sternal or cervical lymph nodes $[2,11,14,17,19]$ that have instead proposed CT as a modality of investigation, and recently, CT was also proposed for morphological and morphometric evaluation of normal abdominal lymph nodes (ALs) to reduce the limitations of ultrasound [5]. Until recently, characteristics studied by CT of the ALs have been described only in relation to specific abdominal pathologies [2]. This study includes several adult dogs that did not show clinical signs attributable to lymphadenopathy and considers the 
examination of normal lymph nodes; as in a previous review [2], the dogs were selected to respond to precise inclusion criteria. In addition to these features, inclusion criteria were restricted starting from the selection of dogs without any alteration in parameters of the blood count and biochemical examination. At least two jejunal lymph nodes, both medial iliac lymph nodes, at least one portal lymph node, the gastric lymph node, the splenic lymph node and the duodenal pancreatic lymph node were identified in almost all dogs. Considering limited information in the literature, the aim of this retrospective study was to establish the normal AL size in presumably healthy dogs according to body weight and age so that this size can provide a reference for evaluation of infectious or neoplastic events. Other secondary objectives were to increase knowledge regarding the characteristics (shape, attenuation and enhancement) of these structures in CT.

\section{Materials And Methods}

The study protocol did not require official or institutional ethical approval as it was not experimental. Owners were properly informed and written consent was obtained for each procedure.

\section{Animals}

Data from all subjects who underwent CT total body in direct scanning and after contrast somministration at the Policlinico Veterinario Roma Sud between December 2014 and December 2017 were analysed. Dogs were selected for analysis according to the following inclusion criteria: achievement of the eighteenth month of life; absence of alterations compatible with an inflammatory or neoplastic process involving the abdomen, pelvis, lower limbs, abdominal or perineum wall; absence of malignant or multicentric processes on the remaining parts of the body with the possibility that metastases could be found in the abdominal organs, pelvis, lower limbs, abdominal or perineum wall; absence of pleural and/or abdominal effusion; absence of movements and/or breathing artefacts; a BCS (body condition score) between $2 / 5$ and 4/5; regarding traumatized subjects, only if less than 12 hours had elapsed since the traumatic event; absence of alterations to blood and urine tests; and absence of anti-inflammatory therapies in the previous 10 days. Standard CVRS laboratory values were used as a reference for blood count and for the following blood chemistry examination parameters: albumin, ALKP, ALT, AST, CPK, GGt, amylase, lipase, BUN, creatinine, phosphorus, calcium, cholesterol, glucose, bilirubin, total proteins and globulins.

\section{Experimental design}

Dogs were divided into three categories (including 15 specimens each) according to body weight as in a previous review for some dimensional evaluations of the small abdominal structure compared to the dog weight [23]: the "S" group consisted of dogs weighing less than or equal to $10 \mathrm{~kg}$; the "M" group consisted of animals with a weight between 10 and $30 \mathrm{~kg}$; and the "L" group consisted of dogs with a weight greater than or equal to $30 \mathrm{~kg}$. As far as age was concerned, dogs were dichotomized into "youths" (dogs aged between 18 and 24 months) and "adults" (dogs aged more than two years). 


\section{Procedures}

The CT procedures were performed under general anaesthesia obtained through Fentanyl ${ }^{\circ}(0.1 \mathrm{mg} / \mathrm{kg} \mathrm{IV})$ as premedication, propofol (5-6.5 mg/ $\mathrm{kg} \mathrm{IV}$ ) for induction and isoflurane administered via gaseous endotracheal tube for maintenance. Transient apnoea was then induced by approximately 60 seconds of hyperventilation and a bolus of fentanyl before each scan. For all subjects, tomographic images were obtained through the use of a 16-slice CT and through the use of the standard logarithm for the acquisition of both direct scans and post-administration of the contrast medium. Contrast medium $(600 \mathrm{mg} / \mathrm{kg} \mathrm{IV}$ ) was injected via an injector at a rate of $3 \mathrm{~mL} / \mathrm{s}$ through $20-22$ gauge intravenous catheters on the right or left cephalic vein. Dogs lay the sternal decubitus with the anterior and posterior limbs extended; the scan parameters were $120 \mathrm{kV}, 190 \mathrm{~mA}, 1-2 \mathrm{~mm}$ slice thickness, pitch of 1:1 and $0.6 \mathrm{~s} /$ rotation. Measurement values were calculated using a soft tissue window with a width of 350 (WW, window width) and a level of 40 (WL, window level).

\section{Measurements}

The ALs analysed were two jejunal, one hepatic, one splenic, one gastric, one pancreaticoduodenal and two medial iliacs. For each lymph node, the localization was recorded by a single observer (S.T.) based on the anatomical indications present in the literature [6,24], and subsequently, a second observer (MV) oversaw and gave consent for data obtained. Dimensions (length, thickness and width), the volume and $\mathrm{X}$-ray attenuations were evaluated.

The length was defined as lymph node's maximum size, regardless of position. The thickness was calculated by measuring the extension in a dorsoventral direction perpendicular to the length. The width was calculated by measuring extension in a laterolateral sense always perpendicular to the length. Software was used to estimate the AL total volume through slice selected areas. The area was calculated on transverse images by the perimeter of a selected structure. This calculation was performed on every single consecutive image that included the selected structure. The perimeter was defined manually. Finally, shapes were recorded by stratifying ALs into three categories: a) elongated, b) roundish, and c) mixed. Shape was judged in a subjective way through 3D volume rendering reconstructions with an evaluation similar to that reported for ultrasonography [18]. Bilobated or multilobed lymph nodes were considered to be elongated. When they could not be clearly entered in one of the two categories, they were categorized as having mixed shape.

Attenuation was measured on the same transverse image, before and after contrast administration, using an oval or round region of interest (ROI) delineated as extensively as possible. Immediately afterwards, if enhancement could distinguish between cortical and medullary areas, the structure was defined as heterogeneous. When contrast was distributed uniformly throughout the entire structure, the enhancement was described as homogeneous.

\section{Statistical analysis}


The mean, standard deviation (SD), median and range (minimum and maximum) were used to describe scalar variables, while absolute and relative frequencies were reported for discrete variables. Nonparametric statistics were applied in order to compare scalar variables (Kruskal-Wallis test), while chi squared tests were used for the analysis of discrete variables.

Data were managed and analysed by using the SPSS Statistics package (IBM Co., Armonk, NY, USA), and two-tailed $P$ values less than 0.05 were considered statistically significant.

\section{Results}

From the archive, 122 apparently healthy dogs were identified based on the CT examination, but only 45 of these dogs were chosen based on the completeness of the medical records, which fully satisfied the inclusion/exclusion criteria during the enrolment period and therefore presumably corresponded to a negative result for abdominal lymphadenopathies.

The indications for CT were 34 dogs with acute thoraco-lumbar spine injury as discopathies or traumatic events (subluxation, luxation or fracture); 4 dogs with Horner's syndrome were negative. In addition, 7 dogs were examined for follow-up six months after surgery.

Dogs were divided into three categories, including 15 specimens each, based on body weight. The "S" group (dogs weighing less than or equal to $10 \mathrm{~kg}$ ) showed an average weight of $6.3 \pm 2.3 \mathrm{~kg}$ (range 2$9 \mathrm{~kg}$ ); the "M" group (animals with a weight between 10 and $30 \mathrm{~kg}$ ) had an average weight of $20.5 \pm 6 \mathrm{~kg}$ (range 12-29 kg); and finally, the "L" group (dogs with a weight greater than or equal to $30 \mathrm{~kg}$ ) had an average weight of $37.9 \pm 8.7 \mathrm{~kg}$, range $30-62 \mathrm{~kg}$ ).

The average total age was $6.2 \pm 3.7$ years (range 1.5-13 years), with overlapping results between various weight categories. Twelve (26.7\%) dogs were "youths" (dogs aged between 18 and 24 months), and 33 (73.3\%) were "adults" (dogs aged more than two years). The average age for the "S" group was $7.5 \pm$ 3.9 years (youths $3 / 15,20.0 \%$, adults $12 / 15,80.0 \%$ ), for the " $M$ " group it is $6.3 \pm 3,7$ years (youths $3 / 15$, $20.0 \%$, adults $12 / 15,80.0 \%$ ), for the $L$ group it was $4.7 \pm 3.0$ years (youths $6 / 15,40.0 \%$, adults $9 / 15$, $60.0 \%)$. No significant differences among the groups were noted $(p=0.36)$.

Out of the 45 dogs selected, 32 ( $71.1 \%)$ were males ( 6 neutered), and $13(28.9 \%)$ were females (1 neutered). There were 9 males and 6 females in the "S" group, among which 1/15 (6.7\%) were sterilized animals; in the " $\mathrm{M}$ " group, there were 10 males and 5 females, among which 2/15 (13.3\%) were sterilized; in the "L" group, there were 13 males and 2 females, among which 4/15 (26.7\%) were sterilized. Additionally, regarding sex, a homogeneous distribution between the weight groups was noted.

The study included mixed-breed dogs $(n=10)$, English cocker spaniels $(n=3)$, Labrador retrievers $(n=3)$, Jack Russell terriers $(n=2)$, Maltese $(n=2)$, dachshunds $(n=2)$, Rottweilers $(n=2)$, Weimaraners $(n=2)$, boxers $(n=2)$, a poodle $(n=1)$, a West Highland white terrier $(n=1)$, a German Pinscher $(n=1)$, a Chihuahua $(n=1)$, a Cavalier King Charles spaniel $(n=1)$, a border collie $(n=1)$, an Irish setter $(n=1)$, a 
beagle $(n=1)$, a Basenji $(n=1)$, a German shepherd $(n=1)$, a course retriever $(n=1)$, a dalmatian $(n=1)$, a Dogo Argentino $(n=1)$, a golden retriever $(n=1)$, a Dobermann $(n=1)$, and a flat coated retriever $(n=1)$.

Four-hundreds and two ALs (out of the total of 405 considered ALs) were visualized and measured, exhibiting positioning abnormalities and variability already described by Beukers et al.; 3 lymph nodes (one hepatic in the $L$ category, one splenic in the $L$ category and one gastric in the $S$ category) were not found. Almost all of the liver lymph nodes were visualized near the hepatic hile (Fig. 1), often symmetrically distributed with one to the right and one to the left of the portal vein. In some cases, two lymph nodes were found along the portal vessel, both on the same side, while in one case, it was not possible to identify more than one. Splenic lymph nodes were usually found along the dorsal margin of the splenic vein (Fig. 2), and almost all of them were disposed with their major axis along the subject's transverse plane rather than on the sagittal plane, as most ALs have been explored. All gastric lymph nodes were found medially with respect to the small gastric curvature (Fig. 3 ) in the passage area between the body and pylorus. A pancreaticoduodenal lymph node was always found in the right pancreatic lobe region (Fig. 4). At least two medial iliac lymph nodes were visualized and measured in each subject, one on the right and one on the left (Fig. 5). These were located at the level of aortic trifurcation caudal to the emergence of the deep iliac circumflex artery. They were usually in a dorsallateral position with respect to the external iliac artery. Finally, at least two jejunal lymph nodes were visualized and measured for each subject and were usually found along the course of the artery or cranial mesenteric vein (Fig. 6).

\section{Lymph node size (length, thickness, width) and volume}

Table 1 shows measurements regarding the length, thickness, width and volume of the AL lymph nodes according to weight categories. 
Table 1

ALs size. Data are reported as mean \pm standard deviation, as well as median and range within parentheses.

\begin{tabular}{|c|c|c|c|c|}
\hline & Length (mm) & $\begin{array}{l}\text { Thickness } \\
(\mathrm{mm})\end{array}$ & Width (mm) & Volume $\left(\mathrm{mm}^{3}\right)$ \\
\hline Hepatic: Total $(n=89)$ & $\begin{array}{l}21.7 \pm 12.2 \\
19.4(4.5- \\
59.7)\end{array}$ & $\begin{array}{l}5.1 \pm 2.0 \\
4.9(1.9- \\
11.2)\end{array}$ & $\begin{array}{l}5.3 \pm 1.8 \\
5.3(2.0- \\
10.7)\end{array}$ & $\begin{array}{l}393 \pm 343 \\
313(12-1,753)\end{array}$ \\
\hline Small: $<10 \mathrm{~kg}(\mathrm{n}=30)$ & $\begin{array}{l}13.8 \pm 6.7 \\
13.8(4.5- \\
33.0)\end{array}$ & $\begin{array}{l}3.6 \pm 1.0 \\
3.5(1.9-5.6)\end{array}$ & $\begin{array}{l}3.8 \pm 1.2 \\
3.5(2.0- \\
6.2)\end{array}$ & $\begin{array}{l}188 \pm 186 \\
146(12-728)\end{array}$ \\
\hline $\begin{array}{l}\text { Medium: } 10-30 \mathrm{~kg}(\mathrm{n}=30) \\
\text { P vs. Small }\end{array}$ & $\begin{array}{l}22.8 \pm 10.5 \\
21.0(5.8- \\
56.8) \\
<0.001\end{array}$ & $\begin{array}{l}5.0 \pm 1.6 \\
4.8(2.5-8.2) \\
0.001\end{array}$ & $\begin{array}{l}5.4 \pm 1.2 \\
5.5(2.8- \\
8.3) \\
<0.001\end{array}$ & $\begin{array}{l}366 \pm 222 \\
360(28-1,028) \\
<0.001\end{array}$ \\
\hline $\begin{array}{l}\text { Large: }>30 \mathrm{~kg}(\mathrm{n}=29) \\
\text { P vs. Small } \\
\text { P vs. Medium }\end{array}$ & $\begin{array}{l}28.8 \pm 13.6 \\
27.3(7.3- \\
59.7) \\
<0.001 \\
0.108\end{array}$ & $\begin{array}{l}6.7 \pm 1.9 \\
6.2(4.4- \\
11.2) \\
<0.001 \\
0.001\end{array}$ & $\begin{array}{l}6.8 \pm 1.8 \\
6.8(3.7- \\
10.7) \\
<0.001 \\
0.003\end{array}$ & $\begin{array}{l}634 \pm 423 \\
520(105-1,753) \\
<0.001 \\
0.015\end{array}$ \\
\hline Overall $P$ value ${ }^{d}$ & $<0.001$ & $<0.001$ & $<0.001$ & $<0.001$ \\
\hline Splenic: Total $(n=44)$ & $\begin{array}{l}14.5 \pm 5.6 \\
14.7(5.1- \\
25.1)\end{array}$ & $\begin{array}{l}5.5 \pm 2.5 \\
4.5(2.1- \\
13.7)\end{array}$ & $\begin{array}{l}5.8 \pm 2.4 \\
5.1(2.7- \\
15.6)\end{array}$ & $\begin{array}{l}256 \pm 206 \\
200(27-967)\end{array}$ \\
\hline Small: $<10 \mathrm{~kg}(\mathrm{n}=15)$ & $\begin{array}{l}10.4 \pm 4.4 \\
9.5(5.1- \\
17.0)\end{array}$ & $\begin{array}{l}3.8 \pm 0.8 \\
3.9(2.1-5.6)\end{array}$ & $\begin{array}{l}4.4 \pm 1.0 \\
4.1(2.9- \\
7.2)\end{array}$ & $\begin{array}{l}89 \pm 67 \\
67(27-294)\end{array}$ \\
\hline $\begin{array}{l}\text { Medium: } 10-30 \mathrm{~kg}(\mathrm{n}=15) \\
\text { P Medium vs. Small }\end{array}$ & $\begin{array}{l}15.8 \pm 5.5^{\mathrm{a} 3} \\
15.3 \pm(8.8- \\
25.1) \\
0.016\end{array}$ & $\begin{array}{l}5.4 \pm 2.0^{\text {a2 }} \\
5.0\left(3.3^{-}\right. \\
10.4) \\
0.051\end{array}$ & $\begin{array}{l}5.7 \pm 2.0^{\text {a3 }} \\
5.7(2.2- \\
10.2) \\
0.065\end{array}$ & $\begin{array}{l}237 \pm 93 \text { a3 } \\
214(111-482) \\
<0.001\end{array}$ \\
\hline
\end{tabular}

Significant $P$ values are highlited by using bold characters ${ }^{a 1} \mathrm{P}<0.05$, ${ }^{\text {a2 }} \mathrm{P}<0.01$ e $\mathrm{e}^{\mathrm{a} 3} \mathrm{P}<0.001$; vs. Small $(<10 \mathrm{~kg})$. Kruskal-Wallis test ${ }^{b 1} \mathrm{P}<0.05,{ }^{b 2} \mathrm{P}<0.01 \mathrm{e}^{\mathrm{b} 3} \mathrm{P}<0.001 ;$ vs. Medium ( $\left.<10 \mathrm{~kg}\right)$. Kruskal-Wallis test ${ }^{\mathrm{d}} \mathrm{P}$ value among the 3 groups (Small, Medium, Large) ; Kruskal-Wallis test 


\begin{tabular}{|c|c|c|c|c|}
\hline & Length (mm) & $\begin{array}{l}\text { Thickness } \\
(\mathrm{mm})\end{array}$ & Width (mm) & Volume $\left(\mathrm{mm}^{3}\right)$ \\
\hline $\begin{array}{l}\text { Large:>30 kg }(n=14) \\
\text { P Large vs. Small } \\
\text { P Large vs. Medium }\end{array}$ & $\begin{array}{l}17.6 \pm 4.4^{\mathrm{a} 3} \\
18.5(11.1- \\
24.2) \\
0.001 \\
0.382\end{array}$ & $\begin{array}{l}7.6 \pm 2.8^{a 3} \text { b2 } \\
7.0(4.5- \\
13.7) \\
<0.001 \\
0.021\end{array}$ & $\begin{array}{l}7.4 \pm 2.9 \text { a3 } \\
b 2 \\
6.9(4.5- \\
15.6) \\
<0.001 \\
0.089\end{array}$ & $\begin{array}{l}454 \pm 229 \text { a3 b1 } \\
435(127-967) \\
<0.001 \\
0.007\end{array}$ \\
\hline Overall $p$ value ${ }^{d}$ & 0.003 & $<0.001$ & 0.001 & $<0.001$ \\
\hline Gastric: Total $(n=44)$ & $\begin{array}{l}9.4 \pm 4 \\
9.6(2.2- \\
19.7)\end{array}$ & $\begin{array}{l}5.7 \pm 2.6 \\
5.1(2.1- \\
13.1)\end{array}$ & $\begin{array}{l}5.9 \pm 2.2 \\
5.7(2.1- \\
11.2)\end{array}$ & $\begin{array}{l}183 \pm 142 \\
157(11-490)\end{array}$ \\
\hline Small:<10 kg $(n=14)$ & $\begin{array}{l}5.5 \pm 2.1 \\
5.5(2.2-9.9)\end{array}$ & $\begin{array}{l}4.1 \pm 1.7 \\
3.8(2.1-7.2)\end{array}$ & $\begin{array}{l}4.6 \pm 1.8 \\
4.8(2.1- \\
6.8)\end{array}$ & $\begin{array}{l}62 \pm 53 \\
36(11-194)\end{array}$ \\
\hline $\begin{array}{l}\text { Medium: } 10-30 \mathrm{~kg}(\mathrm{n}=15) \\
\text { P Medium vs. Small }\end{array}$ & $\begin{array}{l}10.5 \pm 3.0 \\
10.8(5.4- \\
16.0) \\
<0.001\end{array}$ & $\begin{array}{l}6.0 \pm 2.3 \\
5.3(3.8- \\
11.5) \\
0.116\end{array}$ & $\begin{array}{l}5.3 \pm 2.2 \\
5.3(3.8- \\
11.2) \\
0.019\end{array}$ & $\begin{array}{l}195 \pm 115 \\
170(54-450) \\
<0.001\end{array}$ \\
\hline $\begin{array}{l}\text { Large:>30 kg }(n=15) \\
\text { P Large vs. Small } \\
\text { P Large vs. Medium }\end{array}$ & $\begin{array}{l}12.2 \pm 3.4 \\
12.1(5.9- \\
19.7) \\
<0.001 \\
0.146\end{array}$ & $\begin{array}{l}6.8 \pm 3.0 \\
6.5(3.0-13.1) \\
0.014 \\
0.271\end{array}$ & $\begin{array}{l}6.9 \pm 2.1 \\
6.5(3.7- \\
11.0) \\
0.009 \\
0.589\end{array}$ & $\begin{array}{l}284 \pm 144 \\
301(46-490) \\
<0.001 \\
0.110\end{array}$ \\
\hline Overall $p$ value ${ }^{d}$ & $<0.001$ & 0.040 & 0.015 & $<0.001$ \\
\hline $\begin{array}{l}\text { Pancreaticoduodenal: Total } \\
(\mathrm{n}=45)\end{array}$ & $\begin{array}{l}9.9 \pm 4.3 \\
9.1(2.4- \\
24.2)\end{array}$ & $\begin{array}{l}5.9 \pm 2.1 \\
5.8(2.6- \\
10.6)\end{array}$ & $\begin{array}{l}5 \pm 2.1 \\
4.4(1.9- \\
12.6)\end{array}$ & $\begin{array}{l}173 \pm 139 \\
109(12-580)\end{array}$ \\
\hline Small:<10 kg $(n=15)$ & $\begin{array}{l}7.1 \pm 2.2 \\
7.1(2.4- \\
10.1)\end{array}$ & $\begin{array}{l}4.1 \pm 1.3 \\
3.8(2.6-7.8)\end{array}$ & $\begin{array}{l}3.8 \pm 1.2 \\
3.5(1.9- \\
6.2)\end{array}$ & $\begin{array}{l}59 \pm 40 \\
40(12-164)\end{array}$ \\
\hline $\begin{array}{l}\text { Medium: } 10-30 \mathrm{~kg}(\mathrm{n}=15) \\
\text { P Medium vs. Small }\end{array}$ & $\begin{array}{l}10.4 \pm 3.9 \\
9.2(4.4- \\
20.0) \\
0.011\end{array}$ & $\begin{array}{l}6.5 \pm 1.8 \\
6.0(4.3-9.7) \\
<0.001\end{array}$ & $\begin{array}{l}5.0 \pm 1.6 \\
4.4(3.2- \\
9.5) \\
0.040\end{array}$ & $\begin{array}{l}175 \pm 93 \\
214(60-319) \\
<0.001\end{array}$ \\
\hline
\end{tabular}

Significant $P$ values are highlited by using bold characters

${ }^{\text {a1 }} \mathrm{P}<0.05$, ${ }^{\text {a2 }} \mathrm{P}<0.01 \mathrm{e}^{\mathrm{a} 3} \mathrm{P}<0.001$; vs. Small $(<10 \mathrm{~kg})$. Kruskal-Wallis test

b1 $\mathrm{P}<0.05,{ }^{\text {b2 }} \mathrm{P}<0.01 \mathrm{e}^{\mathrm{b} 3} \mathrm{P}<0.001$; vs. Medium $(<10 \mathrm{~kg})$. Kruskal-Wallis test

${ }^{\mathrm{d}} \mathrm{P}$ value among the 3 groups (Small, Medium, Large) ; Kruskal-Wallis test 


\begin{tabular}{|c|c|c|c|c|}
\hline & Length (mm) & $\begin{array}{l}\text { Thickness } \\
(\mathrm{mm})\end{array}$ & Width (mm) & Volume $\left(\mathrm{mm}^{3}\right)$ \\
\hline $\begin{array}{l}\text { Large:>30 kg }(\mathrm{n}=15) \\
\text { P Large vs. Small } \\
\text { P Large vs. Medium }\end{array}$ & $\begin{array}{l}12.4 \pm 5.0 \\
13.0(5.8- \\
24.2) \\
0.002 \\
0.262\end{array}$ & $\begin{array}{l}7.1 \pm 1.8 \\
7.1(3.2- \\
10.6) \\
<0.001 \\
0.280\end{array}$ & $\begin{array}{l}6.4 \pm 2.5 \\
6.6(3.0- \\
12.6) \\
0.002 \\
0.071\end{array}$ & $\begin{array}{l}285 \pm 152 \\
435(74-580) \\
<0.001 \\
0.036\end{array}$ \\
\hline Overall $p$ value ${ }^{d}$ & 0.003 & $<0.001$ & 0.003 & $<0.001$ \\
\hline Medial iliac: Total $(n=90)$ & $\begin{array}{l}25.9 \pm 11 \\
24.6(6-57)\end{array}$ & $\begin{array}{l}5.6 \pm 2.2 \\
5.2(1.9- \\
13.9)\end{array}$ & $\begin{array}{l}5.3 \pm 2 \\
4.9(1.5- \\
11.2)\end{array}$ & $\begin{array}{l}512 \pm 445 \\
354(32-1,987)\end{array}$ \\
\hline Small: $<10 \mathrm{~kg}(\mathrm{n}=30)$ & $\begin{array}{l}17.2 \pm 9.5 \\
16.5(6.0- \\
56.5)\end{array}$ & $\begin{array}{l}3.8 \pm 1.0 \\
3.8(1.9-6.9)\end{array}$ & $\begin{array}{l}3.9 \pm 1.4 \\
4.1(1.5- \\
8.5)\end{array}$ & $\begin{array}{l}194 \pm 170 \\
135(32-713)\end{array}$ \\
\hline $\begin{array}{l}\text { Medium: } 10-30 \mathrm{~kg}(\mathrm{n}=30) \\
\text { P Medium vs. Small }\end{array}$ & $\begin{array}{l}26.8 \pm 7.7 \\
24.5(17.7- \\
55.9) \\
<0.001\end{array}$ & $\begin{array}{l}5.4 \pm 1.8 \\
5.0(2.5- \\
11.1) \\
<0.001\end{array}$ & $\begin{array}{l}5.4 \pm 1.8 \\
5.0(2.5- \\
11.1) \\
<0.001\end{array}$ & $\begin{array}{l}440 \pm 266 \\
214(124-1,272) \\
<0.001\end{array}$ \\
\hline $\begin{array}{l}\text { Large: }>30 \mathrm{~kg}(\mathrm{n}=30) \\
\text { P Large vs. Small } \\
\text { P Large vs. Medium }\end{array}$ & $\begin{array}{l}34.0 \pm 9.1 \\
33.1(20.0- \\
57.0) \\
<0.001 \\
<0.001\end{array}$ & $\begin{array}{l}7.4 \pm 2.4 \\
7.2(4.0- \\
14.0) \\
<0.001 \\
<0.001\end{array}$ & $\begin{array}{l}6.7 \pm 1.9 \\
6.8(3.4- \\
11.2) \\
<0.001 \\
0.007\end{array}$ & $\begin{array}{l}904 \pm 489 \\
844(233-1,987) \\
<0.001 \\
<0.001\end{array}$ \\
\hline Overall $p$ value ${ }^{d}$ & $<0.001$ & $<0.001$ & $<0.001$ & $<0.001$ \\
\hline Jejunal: Total $(n=90)$ & $\begin{array}{l}51.3 \pm 18.3 \\
50(7.7-89.3)\end{array}$ & $\begin{array}{l}5.8 \pm 1.9 \\
5.8(2.4- \\
11.6)\end{array}$ & $\begin{array}{l}6.9 \pm 2.9 \\
6.5(2.3- \\
15.8)\end{array}$ & $\begin{array}{l}1,663 \pm 1,322 \\
1,498(85-5,273)\end{array}$ \\
\hline Small:<10 kg $(n=30)$ & $\begin{array}{l}33.8 \pm 11.1 \\
34.2(7.7- \\
58.1)\end{array}$ & $\begin{array}{l}4.4 \pm 1.4 \\
4.0(2.4-8.0)\end{array}$ & $\begin{array}{l}4.7 \pm 1.6 \\
4.2(2.3- \\
8.1)\end{array}$ & $\begin{array}{l}451 \pm 310 \\
451(85-1,532)\end{array}$ \\
\hline $\begin{array}{l}\text { Medium: } 10-30 \mathrm{~kg}(\mathrm{n}=30) \\
\text { P Medium vs. Small }\end{array}$ & $\begin{array}{l}54.0 \pm 13.2 \\
53.0(28.0- \\
79.0) \\
<0.001\end{array}$ & $\begin{array}{l}6.1 \pm 1.3 \\
5.9(4.1-8.7) \\
<0.001\end{array}$ & $\begin{array}{l}6.8 \pm 2.2 \\
7.0(2.4- \\
11.8) \\
<0.001\end{array}$ & $\begin{array}{l}1709 \pm 979 \\
959(287-3965) \\
<0.001\end{array}$ \\
\hline
\end{tabular}

Significant $P$ values are highlited by using bold characters

${ }^{\text {a1 }} \mathrm{P}<0.05$, ${ }^{\text {a2 }} \mathrm{P}<0.01 \mathrm{e}^{\mathrm{a} 3} \mathrm{P}<0.001$; vs. Small $(<10 \mathrm{~kg})$. Kruskal-Wallis test

b1 $\mathrm{P}<0.05,{ }^{\text {b2 }} \mathrm{P}<0.01 \mathrm{e}^{\mathrm{b} 3} \mathrm{P}<0.001$; vs. Medium $(<10 \mathrm{~kg})$. Kruskal-Wallis test

${ }^{\mathrm{d}} \mathrm{P}$ value among the 3 groups (Small, Medium, Large) ; Kruskal-Wallis test 


\begin{tabular}{|c|c|c|c|c|}
\hline & Length (mm) & $\begin{array}{l}\text { Thickness } \\
(\mathrm{mm})\end{array}$ & Width (mm) & Volume $\left(\mathrm{mm}^{3}\right)$ \\
\hline $\begin{array}{l}\text { Large:>30 kg }(n=30) \\
\text { P Large vs. Small } \\
\text { P Large vs. Medium }\end{array}$ & $\begin{array}{l}66.0 \pm 13.0 \\
65.0(40.9- \\
89.3) \\
<0.001 \\
0.001\end{array}$ & $\begin{array}{l}7.0 \pm 1.9 \\
7.1(3.3- \\
11.6) \\
<0.001 \\
0.029\end{array}$ & $\begin{array}{l}9.2 \pm 2.9 \\
9.2(4.8- \\
15.8) \\
<0.001 \\
0.002\end{array}$ & $\begin{array}{l}2,829 \pm 1,177 \\
2,588(1,028- \\
5,273) \\
<0.001 \\
<0.001\end{array}$ \\
\hline Overall $p$ value ${ }^{d}$ & $<0.001$ & $<0.001$ & $<0.001$ & $<0.001$ \\
\hline \multicolumn{5}{|c|}{ Significant $P$ values are highlited by using bold characters } \\
\hline \multicolumn{5}{|c|}{ a1 $\mathrm{P}<0.05$, a2 $\mathrm{P}<0.01 \mathrm{e}^{\mathrm{a} 3} \mathrm{P}<0.001$; vs. Small $(<10 \mathrm{~kg})$. Kruskal-Wallis test } \\
\hline \multicolumn{5}{|c|}{ b1 $\mathrm{P}<0.05,{ }^{\mathrm{b} 2} \mathrm{P}<0.01 \mathrm{e}^{\mathrm{b} 3} \mathrm{P}<0.001$; vs. Medium $(<10 \mathrm{~kg})$. Kruskal-Wallis test } \\
\hline \multicolumn{5}{|c|}{ d P value among the 3 groups (Small, Medium, Large) ; Kruskal-Wallis test } \\
\hline
\end{tabular}

\section{Lymph node shape}

The prevalence of rounded, elongated or mixed shapes was observed both in the whole population and within body weight categories (Table 2). Some lymph nodes demonstrated a clear prevalence of elongated shapes, such as jejunal lymph nodes and medial iliacs $(97.8 \%$ and $92.2 \%$ of cases, respectively), and hepatic lymph nodes, which showed a slightly lower prevalence (66.3\%). In gastric lymph nodes and in duodenal pancreatic lymph nodes, a rounded shape was more represented (prevalence of $77.3 \%$ and $62.2 \%$, respectively) than in the other nodes. The splenic lymph nodes showed a slight prevalence for a mixed shape (43.2\%), similar to that for an elongated shape (38.6\%). The values of the whole population were homogeneously distributed in weight categories, with some gastric lymph nodes that were rounded in $S$ dogs vs. $33.3 \%$ mixed in both $M$ and $L$ dogs $(P=0.049)$. 
Table 2

Distribution of several shapes in the lymph nodes examined according to localization and weight categories.

\begin{tabular}{|c|c|c|c|}
\hline & Rounded & Mixed & Elongated \\
\hline Hepatic $(\mathrm{n}=89)$ & $6(6.7 \%)$ & $24(27.0 \%)$ & $59(66.3 \%)$ \\
\hline $\begin{array}{l}\text { Small: }<10 \mathrm{~kg}(\mathrm{n}=30) \\
\text { Medium: } 10-30 \mathrm{~kg}(\mathrm{n}=30) \\
\text { Large: }>30 \mathrm{~kg}(\mathrm{n}=29)\end{array}$ & $\begin{array}{l}1(3.3 \%) \\
1(3.3 \%) \\
4(13.8 \%)\end{array}$ & $\begin{array}{l}9(30.0 \%) \\
7(23.3 \%) \\
8(27.6 \%)\end{array}$ & $\begin{array}{l}20(66.7 \%) \\
22(73.3 \%) \\
17(58.6 \%)\end{array}$ \\
\hline$P$ value ${ }^{a}$ & 0.419 & & \\
\hline Splenic $(n=44)$ & $8(18.2 \%)$ & $19(43.2 \%)$ & $17(38.6 \%)$ \\
\hline $\begin{array}{l}\text { Small: }<10 \mathrm{~kg}(\mathrm{n}=15) \\
\text { Medium: } 10-30 \mathrm{~kg}(\mathrm{n}=15) \\
\text { Large: }>30 \mathrm{~kg}(\mathrm{n}=14)\end{array}$ & $\begin{array}{l}1(6.7 \%) \\
2(13.3 \%) \\
5(35.7 \%)\end{array}$ & $\begin{array}{l}7(46.7 \%) \\
7(46.7 \%) \\
5(35.7 \%)\end{array}$ & $\begin{array}{l}7(46.7 \%) \\
6(40.0 \%) \\
4(28.6 \%)\end{array}$ \\
\hline$P$ value ${ }^{a}$ & 0.337 & & \\
\hline Gastric $(n=44)$ & $34(77.3 \%)$ & $10(22.7 \%)$ & $0(0.0 \%)$ \\
\hline $\begin{array}{l}\text { Small: }<10 \mathrm{~kg}(\mathrm{n}=14) \\
\text { Medium: } 10-30 \mathrm{~kg}(\mathrm{n}=15) \\
\text { Large: }>30 \mathrm{~kg}(\mathrm{n}=15)\end{array}$ & $\begin{array}{l}14(100.0 \%) \\
10(66.7 \%) \\
10(66.7 \%)\end{array}$ & $\begin{array}{l}0(0.0 \%) \\
5(33.3 \%) \\
5(33.3 \%)\end{array}$ & $\begin{array}{l}0(0.0 \%) \\
0(0.0 \%) \\
0(0.0 \%)\end{array}$ \\
\hline$P$ value ${ }^{a}$ & 0.049 & & \\
\hline Pancreaticoduodenal $(n=45)$ & $28(62.2 \%)$ & $14(31.1 \%)$ & $3(6.7 \%)$ \\
\hline $\begin{array}{l}\text { Small: }<10 \mathrm{~kg}(\mathrm{n}=15) \\
\text { Medium: } 10-30 \mathrm{~kg}(\mathrm{n}=15) \\
\text { Large: }>30 \mathrm{~kg}(\mathrm{n}=15)\end{array}$ & $\begin{array}{l}10(66.7 \%) \\
8(53.3 \%) \\
10(66.7 \%)\end{array}$ & $\begin{array}{l}5(33.3 \%) \\
5(33.3 \%) \\
4(26.7 \%)\end{array}$ & $\begin{array}{l}0(0.0 \%) \\
2(13.3 \%) \\
1(6.7 \%)\end{array}$ \\
\hline$P$ value $^{a}$ & 0.657 & & \\
\hline Medial iliac $(n=90)$ & $0(0.0 \%)$ & $7(7.8 \%)$ & $83(92.2 \%)$ \\
\hline $\begin{array}{l}\text { Small: }<10 \mathrm{~kg}(\mathrm{n}=30) \\
\text { Medium: } 10-30 \mathrm{~kg}(\mathrm{n}=30) \\
\text { Large: }>30 \mathrm{~kg}(\mathrm{n}=30)\end{array}$ & $\begin{array}{l}0(0.0 \%) \\
0(0.0 \%) \\
0(0.0 \%)\end{array}$ & $\begin{array}{l}5(16.7 \%) \\
1(3.3 \%) \\
1(3.3 \%)\end{array}$ & $\begin{array}{l}25(83.3 \%) \\
29(96.7 \%) \\
29(96.7 \%)\end{array}$ \\
\hline$P$ value ${ }^{a}$ & 0.084 & & \\
\hline Jejunal $(n=90)$ & $0(0.0 \%)$ & $2(2.2 \%)$ & $88(97.8 \%)$ \\
\hline $\begin{array}{l}\text { Small: }<10 \mathrm{~kg}(\mathrm{n}=30) \\
\text { Medium: } 10-30 \mathrm{~kg}(\mathrm{n}=30) \\
\text { Large: }>30 \mathrm{~kg}(\mathrm{n}=30)\end{array}$ & $\begin{array}{l}0(0.0 \%) \\
0(0.0 \%) \\
0(0.0 \%)\end{array}$ & $\begin{array}{l}2(6.7 \%) \\
0(0.0 \%) \\
0(0.0 \%)\end{array}$ & $\begin{array}{l}28(93.3 \%) \\
30(100.0 \%) \\
30(100.0 \%)\end{array}$ \\
\hline \multicolumn{4}{|c|}{ Significant $P$ values are highlited by using bold characters } \\
\hline
\end{tabular}




\begin{tabular}{|c|c|c|c|}
\hline & Rounded & Mixed & Elongated \\
\hline$P$ value ${ }^{a}$ & \multicolumn{3}{|l|}{0.129} \\
\hline \multicolumn{4}{|c|}{ Significant $P$ values are highlited by using bold characters } \\
\hline
\end{tabular}

\section{X-ray attenuation and enhancement}

Observations concerning attenuation on totality of ALs $(n=402)$ show a mean value of $28.3 \pm 6.1$ in scans before contrast administration a mean value of $93.3 \pm 14.0$ in scans after intravenous contrast administration. Subsequently, the same measurements were analysed according to AL localizations, and pre- and post-contrast attenuation values were not significantly different among the 3 body weight categories (Table 3). Only pre-contrast attenuation of jejunal lymph nodes showed significant values $(\mathrm{P}<$ $0.001)$. 
Table 3

X-ray attenuation of ALs before and after contrast administration stratified according to localization and body weight categories.

\begin{tabular}{|c|c|c|c|c|}
\hline & Pre MdC & & Post MdC & \\
\hline & Mean \pm SD & Median (range) & Mean \pm SD & Median (range) \\
\hline Hepatic $(n=89)$ & $29.9 \pm 7.0$ & $29(20-49)$ & $93.7 \pm 10.0$ & $94(73-113)$ \\
\hline $\begin{array}{l}\text { Small: }<10 \mathrm{~kg}(\mathrm{n}=30) \\
\text { Medium: } 10-30 \mathrm{~kg}(\mathrm{n}=30) \\
\text { Large: }>30 \mathrm{~kg}(\mathrm{n}=29)\end{array}$ & $\begin{array}{l}28.0 \pm 5.5 \\
31.0 \pm 8.0 \\
30.5 \pm 7.0\end{array}$ & $\begin{array}{l}26(20-40) \\
31.5(20-49) \\
31(20-49)\end{array}$ & $\begin{array}{l}91.0 \pm 10.0 \\
94.5 \pm 10.0 \\
95.0 \pm 9.5\end{array}$ & $\begin{array}{l}93(73-108) \\
95(77-113) \\
95(77-112)\end{array}$ \\
\hline$P$ value & 0.222 & & 0.328 & \\
\hline Splenic $(n=44)$ & $29.1 \pm 5.5$ & $29.5(19-43)$ & $91.9 \pm 12.5$ & $91.5(70-129)$ \\
\hline $\begin{array}{l}\text { Small: }<10 \mathrm{~kg}(\mathrm{n}=15) \\
\text { Medium: } 10-30 \mathrm{~kg}(\mathrm{n}=15) \\
\text { Large: }>30 \mathrm{~kg}(\mathrm{n}=14)\end{array}$ & $\begin{array}{l}27.0 \pm 4.0 \\
31.0 \pm 6.5 \\
28.5 \pm 5.5\end{array}$ & $\begin{array}{l}27(19-33) \\
32(21-43) \\
28.5(19-37)\end{array}$ & $\begin{array}{l}94.0 \pm 15.0 \\
88.5 \pm 9.5 \\
92.0 \pm 13.0\end{array}$ & $\begin{array}{l}96(75-129) \\
91(76-104) \\
90.5(70-118)\end{array}$ \\
\hline$P$ value & 0.191 & & 0.539 & \\
\hline Gastric $(n=44)$ & $25.4 \pm 4.5$ & $24(19-38)$ & $88.3 \pm 8.5$ & $89.5(73-114)$ \\
\hline $\begin{array}{l}\text { Small: }<10 \mathrm{~kg}(\mathrm{n}=14) \\
\text { Medium: } 10-30 \mathrm{~kg}(\mathrm{n}=15) \\
\text { Large: }>30 \mathrm{~kg}(\mathrm{n}=15)\end{array}$ & $\begin{array}{l}23.5 \pm 4.5 \\
25.0 \pm 2.5 \\
27.0 \pm 5.5\end{array}$ & $\begin{array}{l}22(19-37) \\
25(21-31) \\
27(21-38)\end{array}$ & $\begin{array}{l}89.0 \pm 9.5 \\
87.0 \pm 7.0 \\
89.0 \pm 9.0\end{array}$ & $\begin{array}{l}88(77-114) \\
14(75-101) \\
89(73-102)\end{array}$ \\
\hline$P$ value & 0.079 & & 0.748 & \\
\hline Pancreaticoduodenal $(n=45)$ & $27.0 \pm 5.0$ & $27(19-38)$ & $91.8 \pm 11.0$ & $90(71-129)$ \\
\hline $\begin{array}{l}\text { Small: }<10 \mathrm{~kg}(\mathrm{n}=15) \\
\text { Medium: } 10-30 \mathrm{~kg}(\mathrm{n}=15) \\
\text { Large: }>30 \mathrm{~kg}(\mathrm{n}=15)\end{array}$ & $\begin{array}{l}26.0 \pm 4.0 \\
28.5 \pm 5.0 \\
27.5 \pm 5.5\end{array}$ & $\begin{array}{l}28(20-35) \\
27(21-38) \\
27(19-37)\end{array}$ & $\begin{array}{l}96.0 \pm 10.5 \\
90.0 \pm 8.0 \\
88.0 \pm 14.0\end{array}$ & $\begin{array}{l}97(79-121) \\
91(78-103) \\
87(71-129)\end{array}$ \\
\hline$P$ value & 0.558 & & 0.071 & \\
\hline Medial iliac $(n=90)$ & $27.8 \pm 5.0$ & $27(19-43)$ & $91.8 \pm 11.5$ & $89.5(70-147)$ \\
\hline $\begin{array}{l}\text { Small: }<10 \mathrm{~kg}(\mathrm{n}=30) \\
\text { Medium: } 10-30 \mathrm{~kg}(\mathrm{n}=30) \\
\text { Large: }>30 \mathrm{~kg}(\mathrm{n}=30)\end{array}$ & $\begin{array}{l}26.0 \pm 3.0 \\
29.0 \pm 6.0 \\
28.0 \pm 6.0\end{array}$ & $\begin{array}{l}27(20-32) \\
29(19-41) \\
26.5(19-43)\end{array}$ & $\begin{array}{l}92.5 \pm 11.0 \\
93.0 \pm 18.0 \\
92.0 \pm 19.0\end{array}$ & $\begin{array}{l}92.5(70-108) \\
89(71-147) \\
93(33-132)\end{array}$ \\
\hline$P$ value & 0.145 & & 0.910 & \\
\hline Jejunal $(n=90)$ & $28.7 \pm 6.5$ & $28(19-48)$ & $98.0 \pm 16.5$ & $98.0(70-156)$ \\
\hline
\end{tabular}

Significant $P$ values are highlited by using bold characters

${ }^{\mathrm{d}} \mathrm{P}$ value among the 3 groups ( $\mathrm{S}$ vs. M vs. L) ; Kruskal-Wallis test 


\begin{tabular}{|c|c|c|c|c|}
\hline & Pre MdC & & Post MdC & \\
\hline $\begin{array}{l}\text { Small: }<10 \mathrm{~kg}(\mathrm{n}=30) \\
\text { Medium: } 10-30 \mathrm{~kg}(\mathrm{n}=30) \\
\text { Large: }>30 \mathrm{~kg}(\mathrm{n}=30)\end{array}$ & $\begin{array}{l}25.0 \pm 3.0 \\
32.0 \pm 7.5 \\
29.0 \pm 6.0\end{array}$ & $\begin{array}{l}26(20-32) \\
32(21-48) \\
28.5(19-42)\end{array}$ & $\begin{array}{l}96.0 \pm 12.0 \\
104.0 \pm 22.5 \\
94.0 \pm 11.5\end{array}$ & $\begin{array}{l}96(71-120) \\
102(70-156) \\
95.5(77-121)\end{array}$ \\
\hline$P$ value & $<0.001$ & & 0.121 & \\
\hline \multicolumn{5}{|c|}{ Significant $P$ values are highlited by using bold characters } \\
\hline
\end{tabular}

Regarding the distribution of the contrast medium, AL enhancement according to localization and body weight categories exhibited slightly higher percentages for homogeneous distribution, but no data proved significant (Table 4). 
Table 4

ALs enhancement according to localization and body weight categories.

\begin{tabular}{|c|c|c|}
\hline & \multicolumn{2}{|l|}{ Enhancement } \\
\hline & Homogeneus & Heterogeneous \\
\hline Hepatic $(\mathrm{n}=89)$ & $55(61.8 \%)$ & $34(38.2 \%)$ \\
\hline $\begin{array}{l}\text { Small: }<10 \mathrm{~kg}(\mathrm{n}=30) \\
\text { Medium: } 10-30 \mathrm{~kg}(\mathrm{n}=30) \\
\text { Large: }>30 \mathrm{~kg}(\mathrm{n}=29)\end{array}$ & $\begin{array}{l}15(50.0 \%) \\
25(83.3 \%) \\
15(51.7 \%)\end{array}$ & $\begin{array}{l}15(50.0 \%) \\
5(16.7 \%) \\
14(48.3 \%)\end{array}$ \\
\hline$P$ value ${ }^{a}$ & 0.896 & \\
\hline Splenic $(n=44)$ & $30(68.2 \%)$ & $14(31.8 \%)$ \\
\hline $\begin{array}{l}\text { Small: }<10 \mathrm{~kg}(\mathrm{n}=15) \\
\text { Medium: } 10-30 \mathrm{~kg}(\mathrm{n}=15) \\
\text { Large: }>30 \mathrm{~kg}(\mathrm{n}=14)\end{array}$ & $\begin{array}{l}8(53.3 \%) \\
14(93.3 \%) \\
8(57.1 \%)\end{array}$ & $\begin{array}{l}7(46.7 \%) \\
1(6.7 \%) \\
6(42.9 \%)\end{array}$ \\
\hline$P$ value ${ }^{a}$ & 0.788 & \\
\hline Gastric $(n=44)$ & $34(77.3 \%)$ & $10(22.7 \%)$ \\
\hline $\begin{array}{l}\text { Small: }<10 \mathrm{~kg}(\mathrm{n}=14) \\
\text { Medium: } 10-30 \mathrm{~kg}(\mathrm{n}=15) \\
\text { Large }>30 \mathrm{~kg}(\mathrm{n}=15)\end{array}$ & $\begin{array}{l}10(71.4 \%) \\
13(86.7 \%) \\
11(73.3 \%)\end{array}$ & $\begin{array}{l}4(28.6 \%) \\
2(13.3 \%) \\
4(26.7 \%)\end{array}$ \\
\hline$P$ value ${ }^{a}$ & 0.921 & \\
\hline Pancreatico duodenal $(n=45)$ & $34(75.6 \%)$ & $11(24.4 \%)$ \\
\hline $\begin{array}{l}\text { Small: }<10 \mathrm{~kg}(\mathrm{n}=15) \\
\text { Medium: } 10-30 \mathrm{~kg}(\mathrm{n}=15) \\
\text { Large: }>30 \mathrm{~kg}(\mathrm{n}=15)\end{array}$ & $\begin{array}{l}9(60.0 \%) \\
15(100 \%) \\
10(66.7 \%)\end{array}$ & $\begin{array}{l}6(40.0 \%) \\
0(0.0 \%) \\
5(33.3 \%)\end{array}$ \\
\hline$P$ value ${ }^{a}$ & 0.674 & \\
\hline Medial iliac $(n=90)$ & $59(65.6 \%)$ & $31(34.4 \%)$ \\
\hline $\begin{array}{l}\text { Small: }<10 \mathrm{~kg}(\mathrm{n}=30) \\
\text { Medium: } 10-30 \mathrm{~kg}(\mathrm{n}=30) \\
\text { Large }>30 \mathrm{~kg}(\mathrm{n}=30)\end{array}$ & $\begin{array}{l}17(56.7 \%) \\
28(93.3 \%) \\
14(46.7 \%)\end{array}$ & $\begin{array}{l}13(43.3 \%) \\
2(6.7 \%) \\
16(53.3 \%)\end{array}$ \\
\hline$P$ value $^{a}$ & 0.418 & \\
\hline Jejunal $(n=90)$ & $61(67.8 \%)$ & $29(32.2 \%)$ \\
\hline $\begin{array}{l}\text { Small: }<10 \mathrm{~kg}(\mathrm{n}=30) \\
\text { Medium: } 10-30 \mathrm{~kg}(\mathrm{n}=30) \\
\text { Large }>30 \mathrm{~kg}(\mathrm{n}=30)\end{array}$ & $\begin{array}{l}20(66.7 \%) \\
20(66.7 \%) \\
21(70.0 \%)\end{array}$ & $\begin{array}{l}10(33.3 \%) \\
10(33.3 \%) \\
9(33.3 \%)\end{array}$ \\
\hline
\end{tabular}

${ }^{\text {a }} \mathrm{P}$ value among the 3 groups ( $\mathrm{S}$ vs. M vs. L) ; Kruskal-Wallis test 


\begin{tabular}{|lc|}
\hline \multicolumn{1}{|c|}{ Enhancement } \\
\hline P value $^{\text {a }}$ & 0.784 \\
\hline a P value among the 3 groups (S vs. M vs. L) ; Kruskal-Wallis test \\
\hline
\end{tabular}

\section{Age}

Finally, Table 5 shows the effect of age on size and X-ray attenuation. No significant differences between the youth and adult subgroups were found for hepatic, splenic, gastric, and pancreaticoduodenal lymph nodes. As far as the medial iliac lymph nodes of the $L$ group were concerned, younger dogs showed a significantly higher width $(P=0.026)$ and a significantly lower value of $H U$ postcontrast $(P=0.013)$. Both $M$ and $L$ dogs showed jejunal lymph node thickness and width significantly higher in the youths $(P<$ $0.05)$, and the jejunal lymph node volume of $S$ and $L$ dogs was also significantly higher in the youths $(P<$ 0.05). The HU postcontrast of the jejunal lymph nodes of $S$ dogs was significantly higher in the youths ( $P$ $=0.013)$, while the opposite result was found in the $M$ dogs $(P=0.013)$. 
Table 5

Effect of age on ALs size and attenuation according to localization and body weight. Data are reported as mean \pm standard deviation.

\begin{tabular}{|c|c|c|c|c|c|c|c|}
\hline & $\begin{array}{l}\text { Age } \\
\text { (No.) }\end{array}$ & $\begin{array}{l}\text { Length } \\
(\mathrm{mm})\end{array}$ & $\begin{array}{l}\text { Thickness } \\
(\mathrm{mm})\end{array}$ & $\begin{array}{l}\text { Width } \\
(\mathrm{mm})\end{array}$ & $\begin{array}{l}\text { Volume } \\
\left(\mathrm{mm}^{3}\right)\end{array}$ & $\begin{array}{l}\text { HU } \\
\text { pre } \\
\text { MdC }\end{array}$ & $\begin{array}{l}\text { HU } \\
\text { post } \\
\text { MdC }\end{array}$ \\
\hline \multicolumn{8}{|l|}{ Hepatic } \\
\hline $\begin{array}{l}\text { Small: }:<10 \mathrm{~kg} \\
(\mathrm{n}=30)\end{array}$ & $\begin{array}{l}Y(n= \\
6) \\
A(n= \\
24) \\
P( \\
\text { value }\end{array}$ & $\begin{array}{l}14.5 \pm \\
7.0 \\
13.7 \pm \\
6.7 \\
0.697\end{array}$ & $\begin{array}{l}3.6 \pm 1.3 \\
3.6 \pm 1.0 \\
0.836\end{array}$ & $\begin{array}{l}3.7 \pm \\
1.5 \\
3.9 \pm \\
1.1 \\
0.500\end{array}$ & $\begin{array}{l}203 \pm 18 \\
184 \pm 181 \\
0.678\end{array}$ & $\begin{array}{l}28 \pm \\
6 \\
28 \pm \\
6 \\
0.938\end{array}$ & $\begin{array}{l}94 \pm 3 \\
91 \pm \\
11 \\
0.735\end{array}$ \\
\hline $\begin{array}{l}\text { Medium: } 10-30 \mathrm{~kg} \\
(\mathrm{n}=30)\end{array}$ & $\begin{array}{l}Y(n= \\
6) \\
A(n= \\
24) \\
P \\
\text { value }\end{array}$ & $\begin{array}{l}24.3 \pm \\
8.7 \\
22.4 \pm \\
11.0 \\
0.378\end{array}$ & $\begin{array}{l}5.0 \pm 1.3 \\
5.0 \pm 1.6 \\
0.897\end{array}$ & $\begin{array}{l}5.9 \pm \\
0.7 \\
5.2 \pm \\
1.2 \\
0.177\end{array}$ & $\begin{array}{l}369 \pm 145 \\
365 \pm 239 \\
0.641\end{array}$ & $\begin{array}{l}31 \pm \\
10 \\
31 \pm \\
8 \\
0.755\end{array}$ & $\begin{array}{l}99 \pm \\
14 \\
94 \pm 9 \\
0.35\end{array}$ \\
\hline $\begin{array}{l}\text { Large: }>30 \mathrm{~kg} \\
(\mathrm{n}=29)\end{array}$ & $\begin{array}{l}Y(n= \\
11) \\
A(n= \\
18) \\
P \\
\text { value }\end{array}$ & $\begin{array}{l}29.6 \pm \\
16.1 \\
28.4 \pm \\
12.3 \\
0.964\end{array}$ & $\begin{array}{l}7.4 \pm 2.3 \\
6.4 \pm 1.5 \\
0.312\end{array}$ & $\begin{array}{l}7.6 \pm \\
1.7 \\
6.2 \pm \\
1.5 \\
0.051\end{array}$ & $\begin{array}{l}720 \pm 596 \\
581 \pm 278 \\
0.964\end{array}$ & $\begin{array}{l}31 \pm \\
9 \\
30 \pm \\
6 \\
0.964\end{array}$ & $\begin{array}{l}95 \pm 9 \\
95 \pm \\
10 \\
0.928\end{array}$ \\
\hline \multicolumn{8}{|l|}{ Splenic } \\
\hline $\begin{array}{l}\text { Small: }<10 \mathrm{~kg} \\
(\mathrm{n}=15)\end{array}$ & $\begin{array}{l}Y(n= \\
3) \\
A(n= \\
12) \\
P \\
\text { value }\end{array}$ & $\begin{array}{l}10.7 \pm \\
5.7 \\
10.4 \pm \\
4.4 \\
0.773\end{array}$ & $\begin{array}{l}3.9 \pm 1.5 \\
3.8 \pm 0.7 \\
0.885\end{array}$ & $\begin{array}{l}4.2 \pm \\
0.4 \\
4.4 \pm \\
1.1 \\
0.942\end{array}$ & $\begin{array}{l}144 \pm 136 \\
76 \pm 36 \\
0.665\end{array}$ & $\begin{array}{l}26 \pm \\
4 \\
28 \pm \\
5 \\
0.426\end{array}$ & $\begin{array}{l}100 \pm \\
4 \\
94 \pm \\
17 \\
0.278\end{array}$ \\
\hline $\begin{array}{l}\text { Medium: } 10-30 \mathrm{~kg} \\
(\mathrm{n}=15)\end{array}$ & $\begin{array}{l}Y(n= \\
3) \\
A(n= \\
12) \\
P \\
\text { value }\end{array}$ & $\begin{array}{l}18.4 \pm \\
6.6 \\
15.1 \pm \\
5.3 \\
0.247\end{array}$ & $\begin{array}{l}5.0 \pm 1.1 \\
5.4 \pm 2.2 \\
0.885\end{array}$ & $\begin{array}{l}6.0 \pm \\
1.7 \\
5.6 \pm \\
2.1 \\
0.665\end{array}$ & $\begin{array}{l}272 \pm 24 \\
228 \pm 102 \\
0.149\end{array}$ & $\begin{array}{l}26 \pm \\
7 \\
33 \pm \\
6 \\
0.129\end{array}$ & $\begin{array}{l}91 \pm 3 \\
88 \pm \\
11 \\
0.828\end{array}$ \\
\hline
\end{tabular}

Y,: Youths (18-24 months); A: Adults (>2 years)

Significant $P$ values are highlited by using bold characters 


\begin{tabular}{|c|c|c|c|c|c|c|c|}
\hline & $\begin{array}{l}\text { Age } \\
\text { (No.) }\end{array}$ & $\begin{array}{l}\text { Length } \\
(\mathrm{mm})\end{array}$ & $\begin{array}{l}\text { Thickness } \\
(\mathrm{mm})\end{array}$ & $\begin{array}{l}\text { Width } \\
(\mathrm{mm})\end{array}$ & $\begin{array}{l}\text { Volume } \\
\left(\mathrm{mm}^{3}\right)\end{array}$ & $\begin{array}{l}\text { HU } \\
\text { pre } \\
\text { MdC }\end{array}$ & $\begin{array}{l}\text { HU } \\
\text { post } \\
\text { MdC }\end{array}$ \\
\hline $\begin{array}{l}\text { Large: }>30 \mathrm{~kg} \\
(\mathrm{n}=14)\end{array}$ & $\begin{array}{l}Y(n= \\
6) \\
A(n= \\
8) \\
P \\
\text { value }\end{array}$ & $\begin{array}{l}17.7 \pm \\
4.5 \\
17.5 \pm \\
4.6 \\
0.948\end{array}$ & $\begin{array}{l}8.1 \pm 3.3 \\
7.2 \pm 2.5 \\
0.746\end{array}$ & $\begin{array}{l}7.7 \pm \\
4.2 \\
7.1 \pm \\
1.5 \\
0.477\end{array}$ & $\begin{array}{l}468 \pm 183 \\
445 \pm 271 \\
0.606\end{array}$ & $\begin{array}{l}27 \pm \\
6 \\
30 \pm \\
6 \\
0.363\end{array}$ & $\begin{array}{l}88 \pm \\
12 \\
96 \pm \\
13 \\
0.22\end{array}$ \\
\hline \multicolumn{8}{|l|}{ Gastric } \\
\hline $\begin{array}{l}\text { Small: }<10 \mathrm{~kg} \\
(\mathrm{n}=14)\end{array}$ & $\begin{array}{l}Y(n= \\
3) \\
A(n= \\
11) \\
P \\
\text { value }\end{array}$ & $\begin{array}{l}5.3 \pm \\
1.0 \\
5.6 \pm \\
2.4 \\
0.876\end{array}$ & $\begin{array}{l}5.9 \pm 1.4 \\
4.3 \pm 1.8 \\
0.137\end{array}$ & $\begin{array}{l}5.4 \pm \\
1.8 \\
3.7 \pm \\
1.5 \\
0.102\end{array}$ & $\begin{array}{l}113 \pm 83 \\
48 \pm 36 \\
0.186\end{array}$ & $\begin{array}{l}28 \pm \\
8 \\
22 \pm \\
3 \\
0.098\end{array}$ & $\begin{array}{l}96 \pm \\
17 \\
87 \pm 7 \\
0.435\end{array}$ \\
\hline $\begin{array}{l}\text { Medium: } 10-30 \mathrm{~kg} \\
(\mathrm{n}=15)\end{array}$ & $\begin{array}{l}Y(n= \\
3) \\
A(n= \\
12) \\
P \\
\text { value }\end{array}$ & $\begin{array}{l}10.6 \pm \\
3.1 \\
10.4 \pm \\
3.1 \\
0.885\end{array}$ & $\begin{array}{l}5.7 \pm 2.3 \\
6.2 \pm 2.2 \\
0.665\end{array}$ & $\begin{array}{l}5.5 \pm \\
0.4 \\
6.1 \pm \\
2.6 \\
0.664\end{array}$ & $\begin{array}{l}153 \pm 86 \\
205 \pm 121 \\
0.773\end{array}$ & $\begin{array}{l}28 \pm \\
7 \\
26 \pm \\
4 \\
0.236\end{array}$ & $\begin{array}{l}90 \pm 2 \\
98 \pm \\
11 \\
0.051\end{array}$ \\
\hline $\begin{array}{l}\text { Large: }>30 \mathrm{~kg} \\
(\mathrm{n}=15)\end{array}$ & $\begin{array}{l}Y(n= \\
6) \\
A(n= \\
9) \\
P \\
\text { value }\end{array}$ & $\begin{array}{l}13.7 \pm \\
3.2 \\
11.1 \pm \\
3.3 \\
0.194\end{array}$ & $\begin{array}{l}7.5 \pm 2.4 \\
6.5 \pm 1.8 \\
0.479\end{array}$ & $\begin{array}{l}6.5 \pm \\
2.8 \\
7.0 \pm \\
3.3 \\
0.814\end{array}$ & $\begin{array}{l}279 \pm 125 \\
287 \pm 162 \\
0.906\end{array}$ & $\begin{array}{l}28 \pm \\
7 \\
26 \pm \\
4 \\
0.236\end{array}$ & $\begin{array}{l}90 \pm 2 \\
98 \pm \\
11 \\
0.051\end{array}$ \\
\hline \multicolumn{8}{|l|}{ Pancreaticoduodenal } \\
\hline $\begin{array}{l}\text { Small: }<10 \mathrm{~kg} \\
(\mathrm{n}=15)\end{array}$ & $\begin{array}{l}Y(n= \\
3) \\
A(n= \\
12) \\
P \\
\text { value }\end{array}$ & $\begin{array}{l}7.6 \pm \\
1.7 \\
6.9 \pm \\
2.3 \\
0.770\end{array}$ & $\begin{array}{l}4.8 \pm 2.6 \\
3.9 \pm 0.7 \\
0.880\end{array}$ & $\begin{array}{l}4.7 \pm \\
1.6 \\
3.5 \pm \\
1.1 \\
0.240\end{array}$ & $\begin{array}{l}100 \pm 68 \\
49 \pm 26 \\
0.190\end{array}$ & $\begin{array}{l}28 \pm \\
7 \\
26 \pm \\
4 \\
0.460\end{array}$ & $\begin{array}{l}90 \pm 2 \\
98 \pm \\
11 \\
0.210\end{array}$ \\
\hline $\begin{array}{l}\text { Medium: } 10-30 \mathrm{~kg} \\
(\mathrm{n}=15)\end{array}$ & $\begin{array}{l}Y(n= \\
3) \\
A(n= \\
12) \\
P \\
\text { value }\end{array}$ & $\begin{array}{l}9.1 \pm \\
0.7 \\
10.6 \pm \\
4.3 \\
0.772\end{array}$ & $\begin{array}{l}8.6 \pm 1.6 \\
6.1 \pm 1.4 \\
0.300\end{array}$ & $\begin{array}{l}5.5 \pm \\
1.0 \\
4.8 \pm \\
1.7 \\
0.169\end{array}$ & $\begin{array}{l}200 \pm 114 \\
169 \pm 91 \\
0.470\end{array}$ & $\begin{array}{l}26 \pm \\
3 \\
29 \pm \\
6 \\
0.347\end{array}$ & $\begin{array}{l}86 \pm 7 \\
91 \pm 9 \\
0.248\end{array}$ \\
\hline
\end{tabular}

Y,: Youths (18-24 months); A: Adults (>2 years)

Significant $P$ values are highlited by using bold characters 


\begin{tabular}{|c|c|c|c|c|c|c|c|}
\hline & $\begin{array}{l}\text { Age } \\
\text { (No.) }\end{array}$ & $\begin{array}{l}\text { Length } \\
(\mathrm{mm})\end{array}$ & $\begin{array}{l}\text { Thickness } \\
(\mathrm{mm})\end{array}$ & $\begin{array}{l}\text { Width } \\
(\mathrm{mm})\end{array}$ & $\begin{array}{l}\text { Volume } \\
\left(\mathrm{mm}^{3}\right)\end{array}$ & $\begin{array}{l}\text { HU } \\
\text { pre } \\
\text { MdC }\end{array}$ & $\begin{array}{l}\text { HU } \\
\text { post } \\
\text { MdC }\end{array}$ \\
\hline $\begin{array}{l}\text { Large: }>30 \mathrm{~kg} \\
(\mathrm{n}=15)\end{array}$ & $\begin{array}{l}Y(n= \\
6) \\
A(n= \\
9) \\
P \\
\text { value }\end{array}$ & $\begin{array}{l}12.3 \pm \\
5.2 \\
12.3 \pm \\
5.2 \\
0.906\end{array}$ & $\begin{array}{l}7.3 \pm 1.1 \\
12.7 \pm 5.2 \\
0.724\end{array}$ & $\begin{array}{l}5.5 \pm \\
2.2 \\
6.9 \pm \\
2.6 \\
0.443\end{array}$ & $\begin{array}{l}310 \pm 218 \\
269 \pm 99 \\
0.724\end{array}$ & $\begin{array}{l}28 \pm \\
5 \\
27 \pm \\
6 \\
0.594\end{array}$ & $\begin{array}{l}91 \pm 9 \\
88 \pm \\
17 \\
0.262\end{array}$ \\
\hline \multicolumn{8}{|l|}{ Medial iliac } \\
\hline $\begin{array}{l}\text { Small: }<10 \mathrm{~kg} \\
(\mathrm{n}=30)\end{array}$ & $\begin{array}{l}Y(n= \\
6) \\
A(n= \\
24) \\
P \\
\text { value }\end{array}$ & $\begin{array}{l}15.3 \pm \\
7.8 \\
17.7 \pm \\
10.0 \\
0.736\end{array}$ & $\begin{array}{l}3.5 \pm 0.4 \\
3.9 \pm 1.1 \\
0.406\end{array}$ & $\begin{array}{l}4.6 \pm \\
0.6 \\
3.8 \pm \\
1.4 \\
0.077\end{array}$ & $\begin{array}{l}189 \pm 145 \\
195 \pm 178 \\
0.795\end{array}$ & $\begin{array}{l}26 \pm \\
4 \\
26 \pm \\
3 \\
0.979\end{array}$ & $\begin{array}{l}89 \pm \\
15 \\
90 \pm \\
10 \\
0.856\end{array}$ \\
\hline $\begin{array}{l}\text { Medium: } 10-30 \mathrm{~kg} \\
(\mathrm{n}=30)\end{array}$ & $\begin{array}{l}Y(n= \\
6) \\
A(n= \\
24) \\
P \\
\text { value }\end{array}$ & $\begin{array}{l}31.0 \pm \\
14.3 \\
25.7 \pm \\
4.9 \\
0.856\end{array}$ & $\begin{array}{l}5.4 \pm 1.9 \\
8.3 \pm 1.4 \\
0.938\end{array}$ & $\begin{array}{l}5.1 \pm \\
2.3 \\
5.4 \pm \\
1.7 \\
0.716\end{array}$ & $\begin{array}{l}516 \pm 438 \\
420 \pm 214 \\
0.795\end{array}$ & $\begin{array}{l}27 \pm \\
7 \\
30 \pm \\
6 \\
0.286\end{array}$ & $\begin{array}{l}94 \pm \\
22 \\
94 \pm \\
18 \\
0.736\end{array}$ \\
\hline $\begin{array}{l}\text { Large: }>30 \mathrm{~kg} \\
(\mathrm{n}=30)\end{array}$ & $\begin{array}{l}Y(n= \\
12) \\
A(n= \\
18) \\
P \\
\text { value }\end{array}$ & $\begin{array}{l}35.8 \pm \\
9.5 \\
32.4 \pm \\
8.7 \\
0.397\end{array}$ & $\begin{array}{l}7.4 \pm 1.8 \\
7.5 \pm 2.8 \\
0.539\end{array}$ & $\begin{array}{l}7.5 \pm \\
1.0 \\
6.1 \pm \\
2.1 \\
0.026\end{array}$ & $\begin{array}{l}1,088 \pm \\
451 \\
782 \pm 486 \\
0.069\end{array}$ & $\begin{array}{l}26 \pm \\
5 \\
29 \pm \\
7 \\
0.097\end{array}$ & $\begin{array}{l}84 \pm \\
20 \\
97 \pm \\
17 \\
0.013\end{array}$ \\
\hline \multicolumn{8}{|l|}{ Jejunal } \\
\hline $\begin{array}{l}\text { Small: }<10 \mathrm{~kg} \\
(\mathrm{n}=30)\end{array}$ & $\begin{array}{l}Y(n= \\
6) \\
A(n= \\
24) \\
P \\
\text { value }\end{array}$ & $\begin{array}{l}34.8 \pm \\
7.1 \\
33.5 \pm \\
12.0 \\
0.959\end{array}$ & $\begin{array}{l}5.3 \pm 1.5 \\
4.2 \pm 1.3 \\
0.077\end{array}$ & $\begin{array}{l}5.7 \pm \\
1.8 \\
4.4 \pm \\
1.5 \\
0.108\end{array}$ & $\begin{array}{l}738 \pm 471 \\
378 \pm 214 \\
0.043\end{array}$ & $\begin{array}{l}27 \pm \\
4 \\
25 \pm \\
3 \\
0.097\end{array}$ & $\begin{array}{l}102 \pm \\
7 \\
95 \pm \\
13 \\
0.013\end{array}$ \\
\hline $\begin{array}{l}\text { Medium: } 10-30 \mathrm{~kg} \\
(\mathrm{n}=30)\end{array}$ & $\begin{array}{l}Y(n= \\
6) \\
A(n= \\
24) \\
P \\
\text { value }\end{array}$ & $\begin{array}{l}59.4 \pm \\
8.2 \\
52.7 \pm \\
13.9 \\
0.154\end{array}$ & $\begin{array}{l}7.0 \pm 1.1 \\
5.8 \pm 1.2 \\
0.040\end{array}$ & $\begin{array}{l}9.6 \pm \\
1.9 \\
6.1 \pm \\
1.7 \\
0.002\end{array}$ & $\begin{array}{l}2,339 \pm \\
971 \\
1,551 \pm \\
935 \\
0.062\end{array}$ & $\begin{array}{l}27 \pm \\
6 \\
34 \pm \\
7 \\
0.097\end{array}$ & $\begin{array}{l}84 \pm \\
13 \\
109 \pm \\
22 \\
0.013\end{array}$ \\
\hline
\end{tabular}

Y,: Youths (18-24 months); A: Adults (> 2 years)

Significant $P$ values are highlited by using bold characters 


\begin{tabular}{|c|c|c|c|c|c|c|c|}
\hline & $\begin{array}{l}\text { Age } \\
\text { (No.) }\end{array}$ & $\begin{array}{l}\text { Length } \\
(\mathrm{mm})\end{array}$ & $\begin{array}{l}\text { Thickness } \\
(\mathrm{mm})\end{array}$ & $\begin{array}{l}\text { Width } \\
(\mathrm{mm})\end{array}$ & $\begin{array}{l}\text { Volume } \\
\left(\mathrm{mm}^{3}\right)\end{array}$ & $\begin{array}{l}\text { HU } \\
\text { pre } \\
\text { MdC }\end{array}$ & $\begin{array}{l}\text { HU } \\
\text { post } \\
\text { MdC }\end{array}$ \\
\hline $\begin{array}{l}\text { Large: }>30 \mathrm{~kg} \\
(\mathrm{n}=30)\end{array}$ & $\begin{array}{l}Y(n= \\
12) \\
A(n= \\
18) \\
P \\
\text { value }\end{array}$ & $\begin{array}{l}67.5 \pm \\
12.3 \\
65.0 \pm \\
14.3 \\
0.626\end{array}$ & $\begin{array}{l}8.3 \pm 1.7 \\
6.2 \pm 1.6 \\
0.007\end{array}$ & $\begin{array}{l}10.7 \pm \\
2.9 \\
8.2 \pm \\
2.5 \\
0.029\end{array}$ & $\begin{array}{l}3,796 \pm \\
895 \\
2,184 \pm \\
862 \\
<0.001\end{array}$ & $\begin{array}{l}28 \pm \\
5 \\
30 \pm \\
7 \\
0.408\end{array}$ & $\begin{array}{l}94 \pm \\
15 \\
93 \pm 9 \\
0.899\end{array}$ \\
\hline \multicolumn{8}{|c|}{ Y,: Youths (18-24 months); A: Adults (>2 years) } \\
\hline \multicolumn{8}{|c|}{ Significant $P$ values are highlited by using bold characters } \\
\hline
\end{tabular}

\section{Discussion}

One of the main objectives of this research is to study a possible relationship between AL CT measurements that are most visible and body weight in dogs without any sign of lymphadenopathy found through clinical evaluation, basic blood tests and imaging. The population's specimens included had good variability in breed and weight but showed a homogeneous distribution between groups $S, M$ and $L$ regarding different variables, such as age $(P=0.36)$, sex or sterilization $(P=0.25, P=0.31$, respectively). In this ideal population, statistical analysis revealed a significant increase in $\mathrm{AL}$ measurements from a dog group with a lower weight to greater weight. Data obtained from all group comparisons showed statistical significance $(P<0.05)$ for each measurement performed, confirming the initial hypothesis. In anticipation of achieving this result, what is more interesting is which of these measures is more reliable to carry out a dimensional assessment. Although there is good significance for length, thickness and width, for volume, the percentage values were the most reliable $(P<0.001)$. Both for the authors' experience and significance obtained, the possibility of easily calculating the volume is an excellent means for a dimensional evaluation. Here, 3D volume rendering reconstructions made available by various software programs, in addition to expressing the numerical value of the volume, quickly revealed a shape to observers; this technology also allows evaluation outside other abdominal structures that may interfere with interpretation. From a single comparison between weight classes, another interesting analysis result was obtained. Although values were significantly increasing in multiple contracts between all groups, this did not always happen by comparing them to pairs. Hepatic lymph nodes, for example, showed only slight differences $(P>0.05)$ in length values between dogs weighing between 10 and $30 \mathrm{~kg}(\mathrm{M})$ and dogs weighing more than $30 \mathrm{~kg}(\mathrm{~L})$. In splenic tissue instead of width, significant data were obtained $(P<0.001)$ only for comparison between dogs of the $S$ group and those of the $L$ group. Indeed, subjects in the $M$ group were coupled with those in the $S$ group, and the $L$ group revealed greater error possibilities $(P>0.05)$ than in the other groups. The same happened when comparing the length between categories $M$ and $L$ or the thickness between group $S$ and group $M(p>$ 0.05). The gastric lymph nodes showed insignificant data for the thickness between specimens weighing less than $10 \mathrm{~kg}(\mathrm{~S})$ and those weighing between 10 and $30 \mathrm{~kg}(\mathrm{M})$, and no measurement was found to be 
reliable comparing dogs of group $\mathrm{M}$ and those of $\mathrm{L}$. The same outcome also occurred for pancreaticoduodenal lymph nodes, except for the volume $(P<0.05)$. Finally, for the medial iliac and jejunal lymph nodes, significant values were maintained in all measurements $(P<0.05)$ in comparison between the two body weight categories. Certainly, volume size, even by latter estimate, is the most reliable value. Except for gastric lymph nodes, it was the only value that always showed little chance of error $(P<0.05)$.

Taking as population under consideration ALs regardless of location and weight divisions, the results obtained from attenuation and enhancement are very similar to those already present in the literature [5]. However, the goal of interest is to confirm that these characteristics are not actually affected by the body weight. In Group S, M and L ALs grouped by location, there were no values with significant differences ( $P$ $>0.05$ ). Different Hounsfield values in these structures are random, and feedback for ALs is compatible with the classical attenuation values obtained by soft tissue. Regarding enhancement, there are no significant data. The expectation of the authors, for subjects that were presumably normal, was to obtain most of the investigated structures with homogeneous capturing, as described recently for sternal lymph nodes $[9,17]$. In most structures, the contrast medium was distributed homogeneously, but there were also a large number of lymph nodes with a heterogeneous distribution. Regarding this study, it must be remembered that a heterogeneous enhancement is also assigned to those structures in which one could easily observe a distinction between the cortical and medullary regions and therefore not necessarily identify a pathological process in progression expression. Heterogeneous, "ring" or absent enhancement of ALs in CT exams has been described in veterinary medicine $[2,14,25]$ and in the clinic $[7,8,12,20,24]$, usually as an expression of pathological processes such as inflammation or metastasis. Care should be taken, however, because homogenous enhancement is not specific to normality, and heterogeneity is not exclusive to a pathological process. Evaluating only a homogeneous distribution is therefore insufficient; possible enhancement patterns are many, there are mixed patterns, the patterns may be peripheral, the lymph nodes may not acquire contrast to be subsequently normal or may be affected by inflammatory or tumour processes. It is therefore important to evaluate this factor always in relation to other features (for example, shape and size). Additionally, shape evaluation shows few differences from what has already been treated [5]. This work, however, checks whether there is a correlation between the weight and AL length, which then results in an elongated shape. The values obtained were not significant for any of the investigated locations, except for the gastric lymph nodes $(P<0.05)$. The shapes of these structures are therefore in almost all cases an anatomical feature invariant under normal conditions, despite the body weight differences between the examined dogs.

The ultimate goal is to observe measurement behaviour obtained by separating younger from older dogs while maintaining weight differences. One of the inclusion criteria was reaching 18 months of age, so this study did not include puppies. Young subjects are animals that were suspected to be aged between 18 and 24 months. The same measurements described above were used to compare length, thickness, width and volume between these two subgroups (youths vs. adults). The reason for this distinction is to ensure that these subjects have no age-related differences concerning the AL size; therefore, a dog aged 18 months can presumably be considered an adult in the context of CT-guided measurements of some 
ALs. Comparison does not raise any particular significance to many of the ALs involved. Regarding jejunal lymph nodes only, the data conflict with significant values in different weight categories and for different sizes. There seems to be a positive association in dogs under $10 \mathrm{~kg}$ with regard to volume $(\mathrm{P}<$ $0.05)$ and in subjects belonging to category $M$ for thickness and width $(P<0.05)$. In dogs weighing more than $30 \mathrm{~kg}$, positivity was found for thickness, width and volume $(P<0.05)$. For volume, the values are very close to being meaningful for dogs of category $M(P=0.062)$. Therefore, considering volume to be the most reliable metric, it is reasonable to think that there is a positive correlation between subject's young age (18-24 months) and a higher volumetric value only for jejunal lymph nodes. This possibility is also analysed in human medicine; for example, an increase in the mesenteric lymph node size found with CT in paediatric subjects (range 1.1-17.3 years) is considered a non-specific lymphadenopathy finding. Furthermore, for children, a maximum dimensional limit slightly higher than adult subjects is set [10].

In conclusion, we have verified with this research that dogs exhibit some significant changes in the normal abdominal lymph node size according to the body weight. These data can be used in dogs as a reference for size and appearance of hepatic, splenic, gastric, duodenal pancreatic, medial and jejunal iliac lymph nodes in relation to their body weight and thus lay foundations for a comparison with the results obtained from unhealthy subjects. Through these evaluations, we can therefore differentiate with certainty subjects suffering from inflammatory or tumour processes in the abdomen or in other regions. Our proposal is therefore to insert new normal dimensional parameters, catalogued according to the dog size, regarding the length, width, thickness, volume and shape in order to evaluate them in future studies and routine clinical investigations in consideration of a variable such as body weight whose importance cannot be underestimated in veterinary medicine.

\section{Declarations}

\section{Authors contribution}

ST, GA, RT AND MV designed the experiments and wrote the paper; ST, GA, RT, FS and MV the experiments and collected the samples. AML analyzed the data. MV was responsible for the final content. All of the authors have read and approved the final manuscript.

\section{Funding}

This work was not supported by any funding.

\section{Availability of data and materials}

The datasets used and/or analysed during the current study are available from the corresponding author by reasonable request.

\section{Ethics approval and consent to participate}


No ethical approval has been required for this study. This study did not require official or institutional ethical approval as it was not experimental. All dogs in this study were examined with the written consent of their owners.

\section{Consent for publication}

The owners signed an informed consent form including the authorization to publish the data arising from this study.

\section{Competing interests}

The authors declare that they have no competing interests.

\section{References}

1. Agthe P, Caine AR, Posch B, Herrtage ME. Ultrasonographic appearance of jejunal lymph nodes in dogs without clinical signs of gastrointestinal disease. Vet Radiol Ultrasound. 2009; 50(2):195-200.

2. Ballegeer EA, Adams WM, Dubielzig RR, Paoloni MC; Klauer JM, Keuler NS. Computed tomography characteristics of canine tracheobronchial lymph node metastasis. Vet Radiol Ultrasound. 2010;51(4):397-403.

3. Barberet V, Schreurs E, Rademacher N, Nitzl G, Taeymans O, Duchateau L, Saunders JH. Quantification of the effect of various patient and image factors on ultrasonographic detection of select canine abdominal organs. Veterinary Radiology \& Ultrasound, 2008; 49(3):273-276.

4. Bertolini G, Furlanello T, De Lorenzi D, Caldin M. Computed tomographic quantification of canine adrenal gland volume and attenuation. Veterinary Radiology \& Ultrasound. 2006;47(5).444-448.

5. Beukers M, Grosso FV, Voorhout G. Computed tomographic characteristics of presumed normal canine abdominal lymph nodes. Vet Radiol Ultrasound. 2013; 54(6):610-617.

6. Bezuidenhout AJ. The lymphatic system. In: Evans HE, Lahunta de A editor. Miller's anatomy of the dog 4th ed. St. Louis: Elsevier Saunders, 2013.

7. Chen J, Yang ZG, Shao H, Xiao JH, Deng W, Wen LY, Tang SS. Differentiation of tuberculosis from lymphomas in neck lymph nodes with multidetector-row computed tomography. IJTLD. 2012; 16(12):1686-1691.

8. Hagtvedt T, Aaløkken TM, Smith H, Graff BA, Holte H, Kolbenstvedt A. Enhancement character-istics of lymphomatous lymph nodes of the neck. Acta Radiol. 2010; 51(5):475-481.

9. Iwasaki R, Mori T, Ito Y, DVM, Kawabe M, Murakmi M, Maruo K. Computed Tomographic Evaluation of Presumptively Normal Canine Sternal Lymph Nodes. J Am Anim Hosp Assoc. 2016;52(6):371-377.

10. Karmazyn B, Werner EA, Rejaie B, Applegate KE. Mesenteric lymph nodes in children: What is normal? Ped Radiol. 2005; 35:774-7.

11. KneissI S, Probst A. Comparison of computed tomographic images of normal cranial and upper cervical lymph nodes with corresponding e12 plastinated-embedded sections in the dog. The Vet J. 
2007;174(2):435-438.

12. Kwee RM and Kwee TC. Imaging in assessing lymph node status in gastric cancer. J Gastric Cancer, 2009;12(1):6-22.

13. Llabrés-Diaz FJ. Ultrasonography of the medial iliac lymph nodes in the dog. Vet Radiol Ultrasound. 2004;45(2): 156-165.

14. Marolf AJ, Gibbons DS, Podell BK, Park RD. Computed tomographic appearance of primary lung tumors in dogs. Vet Radiol Ultrasound. 2011;45(2):156-165.

15. Mattoon JS, Berry CR, Nyland TG. Advanced Ultrasound Techniques. In: Mattoon JS, Nyland TG, editors. Small animal diagnostic ultrasound 3rd ed. Elsevier, 2015;13:501-516.

16. Mayer MN, Lawson JA, Silver TI. Sonographic characteristics of presumptively normal canine medial iliac and superficial inguinal lymph nodes. Vet Radiol Ultrasound. 2010;51(6):638-641.

17. Miloncev M, Nemanic S, Bobe G. Computed tomographic assessment of sternal lymph node dimensions and attenuation in healthy dogs. Am J Vet Res. 2017;78(3):289-294.

18. Nyman H. Abdominal lymph nodes. In: O'Brien B, Barr F, editors. Manual of Canine and Feline Abdominal Imaging BSAVA, 2008; 7:59-75.

19. Paoloni MC, Adams WM, Dubielzig RR, et al. Comparison of results of computed tomography and radiography with histopathologic findings in tracheobronchial lymph nodes in dogs with primary lung tumors: 14 cases (1999-2002). J Am Vet Med Assoc. 2006; 228(11):1718-1722.

20. Pombo F, Rodriguez E, Caruncho MV, Villalva C, Crespo C. CT attenuation values and enhancing characteristics of thoracoabdominal lymphomatous adenopathies. J Comput Assist Tomog. 1994;18(1):59-62.

21. Pugh CR. Ultrasonographic examination of abdominal lymph nodes in the dog. Vet radiol Ultrasound. 1994;35(2):110-115.

22. Rossi F, Patsikas MN, Wisner ER. Abdominal lymph nodes and lymphatic collecting system. In: Schwarz T, Saunders JH editors. Veterinary computed tomography. 1st ed. Chichester: John Wiley \& Sons Ltd. 2011; 36:371-379.

23. Soulsby SN, Holland M, Hudson JA, Behrend EN. Ultrasonographic evaluation of adrenal gland size compared to body weight in normal dogs. Vet Radiol Ultrasound. 2014;56(3):317-326.

24. Tang SS, Yang ZG, Deng W, Shao H, Chen J, Wen LY. Differentiation between tuberculosis and lymphoma in mediastinal lymph nodes: Evaluation with contrast-enhanced MDCT. Clin Radiol. 2012;67(9):877-883.

25. Tsai S, Sutherland-Smith J, Burgess K, Ruthazer R, Sato A. Imaging characteristics of intrathoracic histiocytic sarcoma in dogs. Vet Radiol Ultrasound. 2012; 53(1):21-27.

\section{Figures}




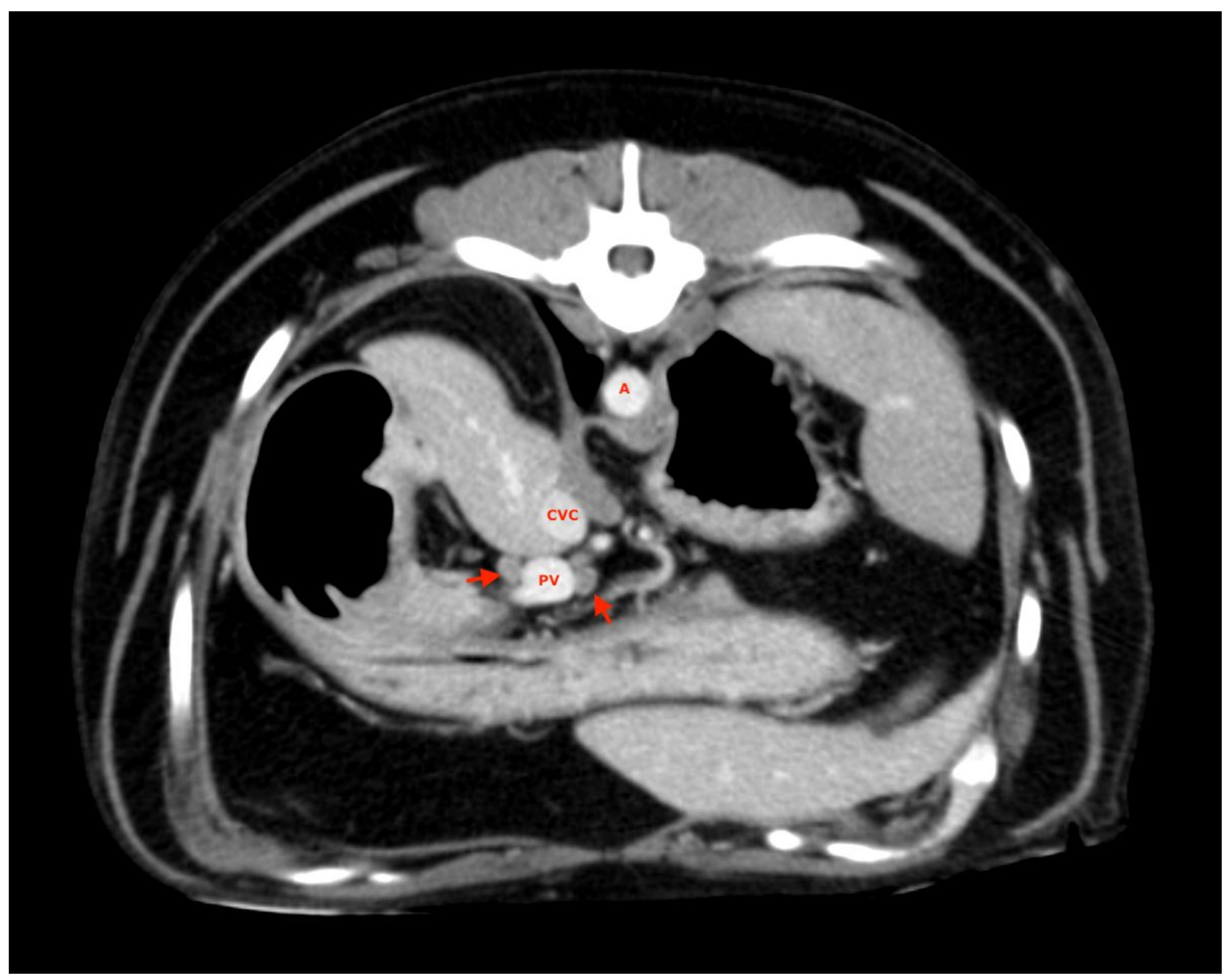

Figure 1

Hepatic lymph nodes. A, aorta; CVC, caudal vena cava; PV, portal vein. 


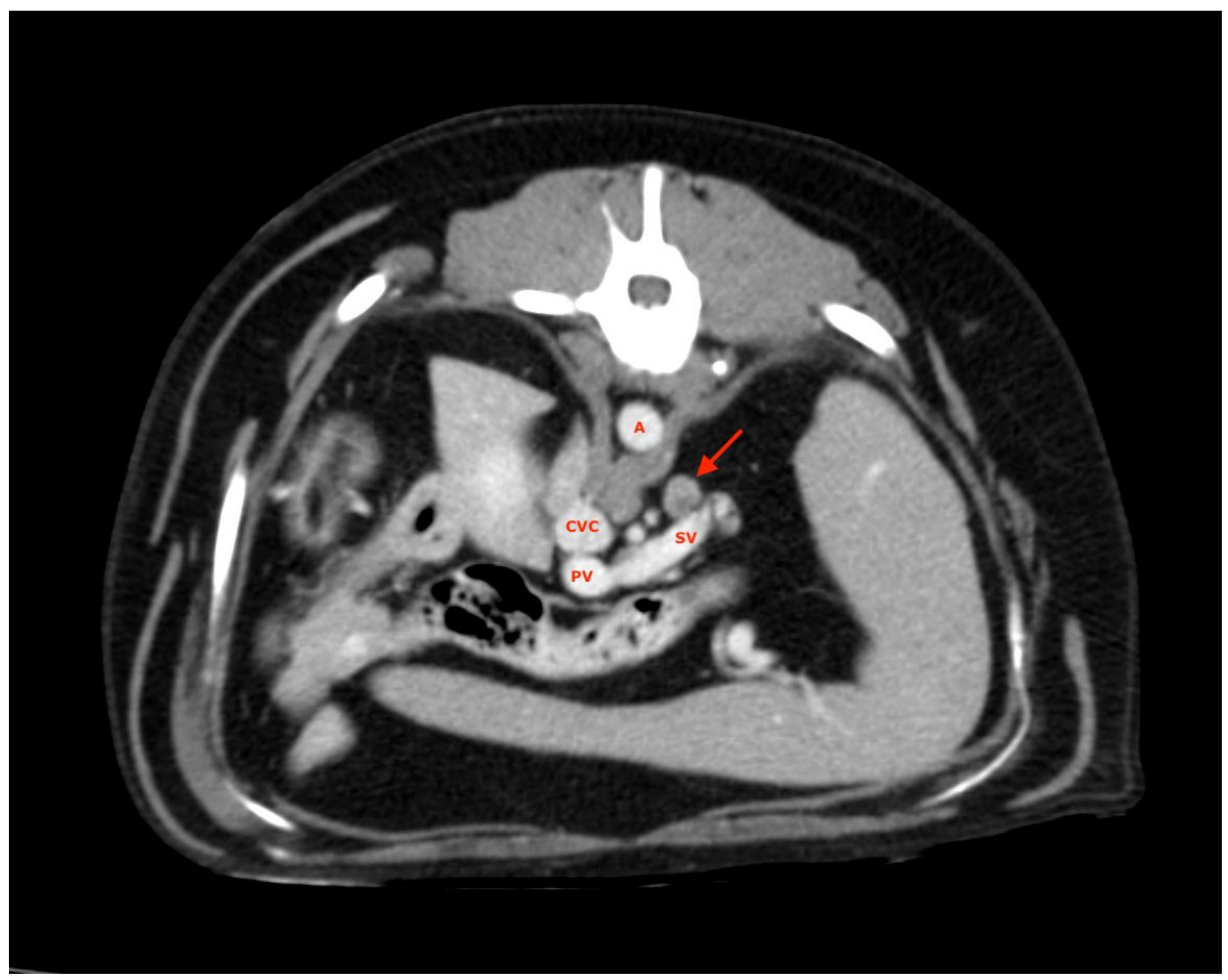

Figure 2

Splenic lymph node. A, aorta; CVC, caudal vena cava; PV, portal vein; SV, splenic vein. 


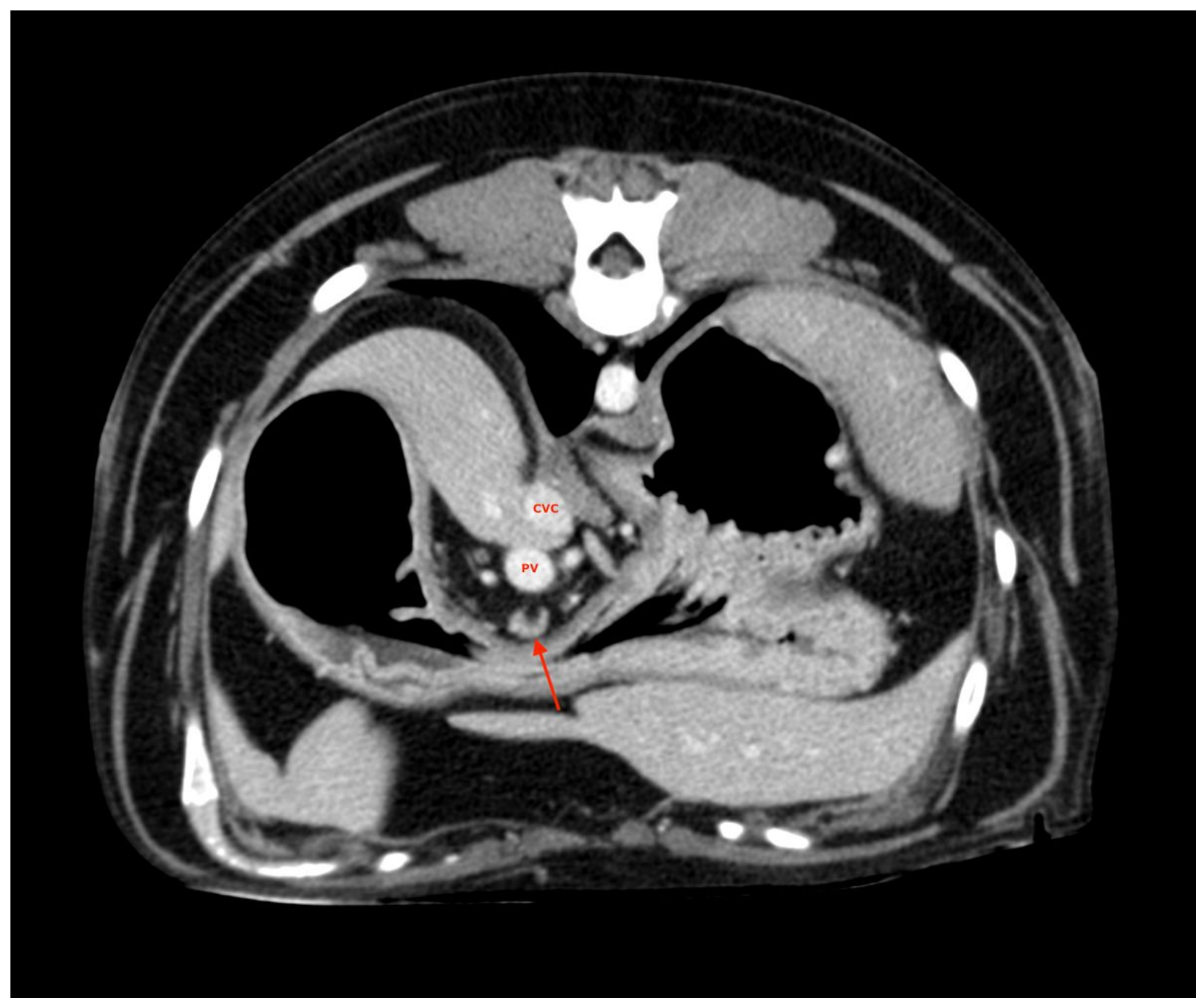

Figure 3

Gastric lymph node. A, aorta; CVC, caudal vena cava. 


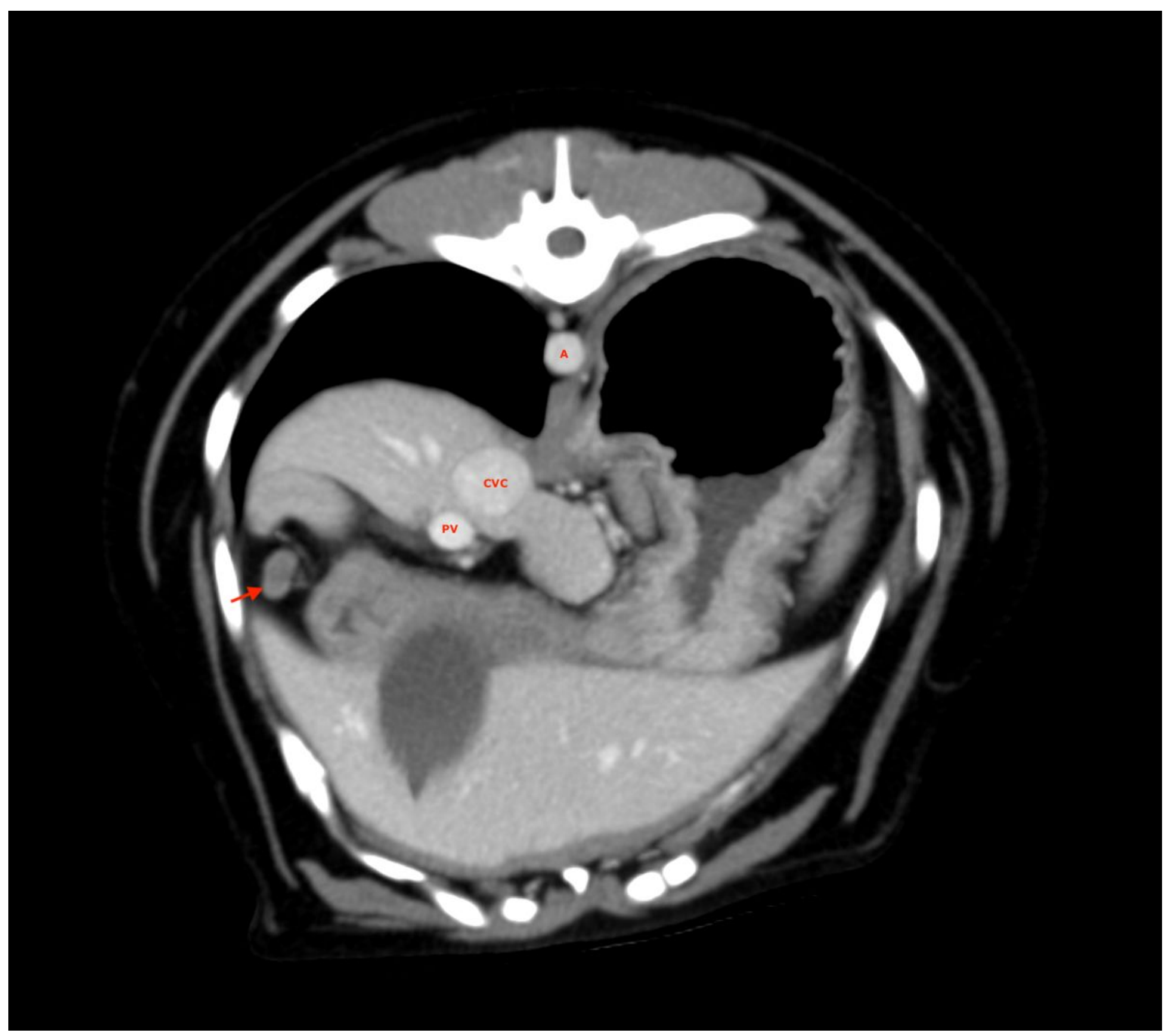

Figure 4

Pancreaticoduodenal lymph node. A, aorta; CVC, caudal vena cava; PV, portal vein. 


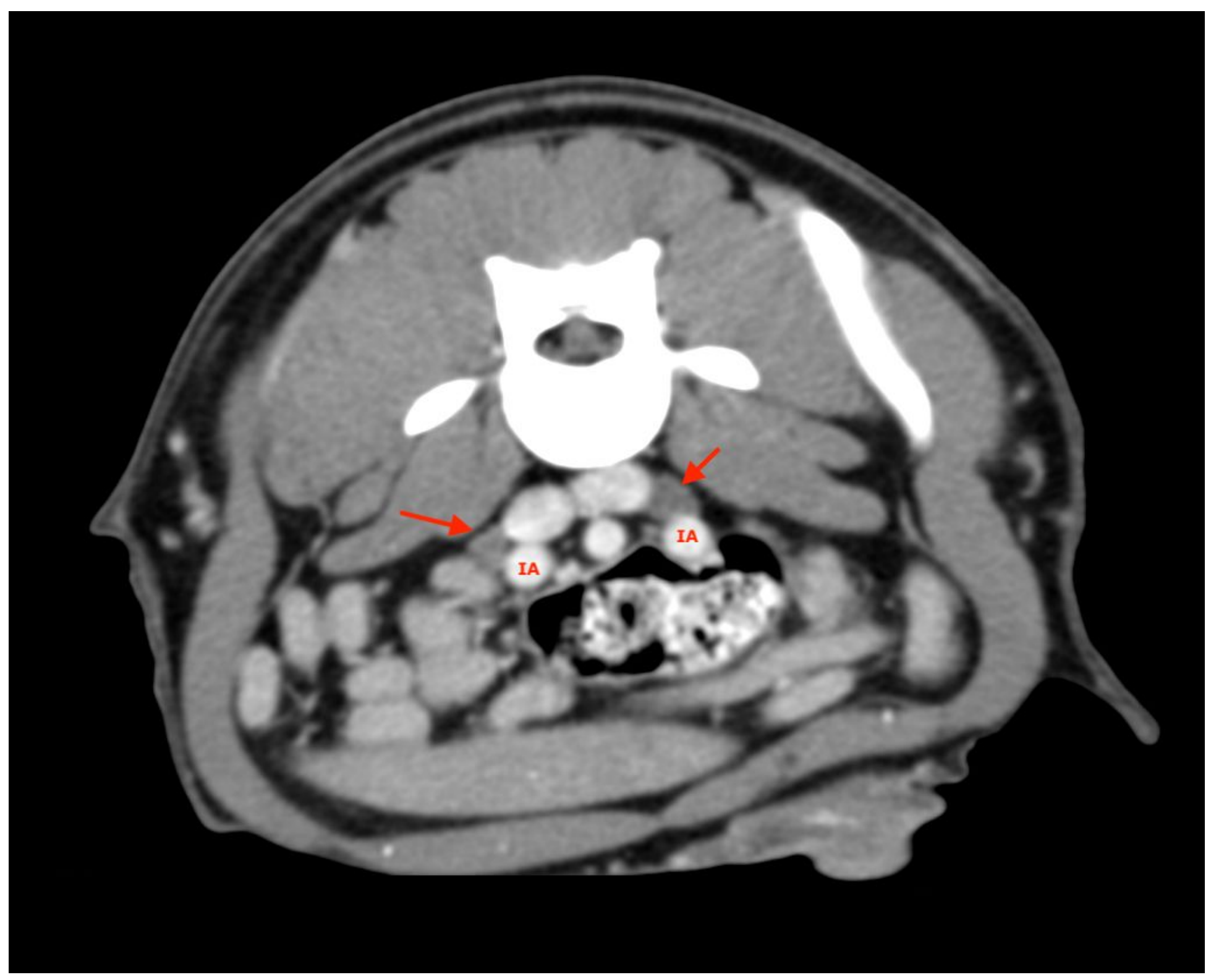

Figure 5

Medial Iliac lymph nodes. IA, iliac artery 


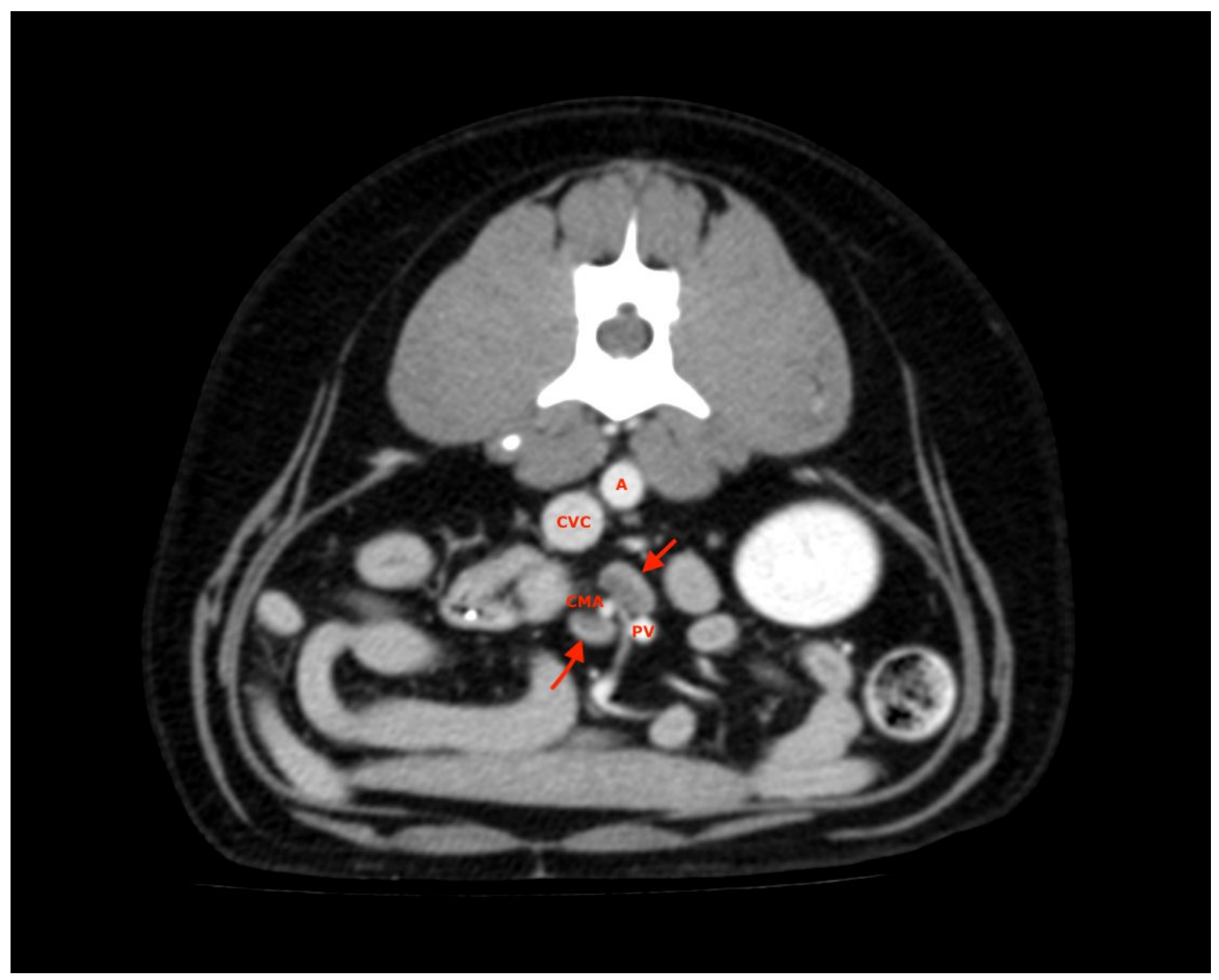

\section{Figure 6}

Jejunal lymph nodes. A, aorta; CVC, caudal vena cava; PV, portal vein; CMA, cranial mesenteric artery. 